\title{
Longitudinal spin relaxation in simple stochastic models for disordered systems
}

\author{
P. Borgs, K.W. Kehr, and P. Heitjans \\ Institut für Festkörperforschung, Forschungszentrum Jülich GmbH, Postfach 1913, 52425 Jülich, Germany \\ and Institut für Physikalische Chemie, Universität Hannover, Callinstrasse 3-3a, 30167 Hannover, Germany
}

(Received 1 August 1994; revised manuscript received 27 December 1994)

\begin{abstract}
The relaxation of single probe spins was investigated for simple models of systems with quenched disorder. The spin relaxation was calculated for a two-site model with arbitrarily oriented magnetic fields and the result was averaged over various distributions of the fields, and of the hopping rates of the spin. On an intermediate time scale, a modified Kubo-Toyabe behavior is obtained for large hopping rates, in agreement with recent $\mu \mathrm{SR}$ experiments. A stretched-exponential decay of the spin polarization is obtained at longer times. The Kohlrausch exponent is found to be field and hopping-rate dependent, in qualitative agreement with recent NMR and $\beta$-NMR experiments. The resulting longitudinal relaxation rate still does not show the significant deviations from the Bloembergen-Purcell-Pound (BPP) behavior that are typical for glassy systems. Therefore, the random two-frequency model was extended to include time-dependent renewals of the environment. This modification may yield asymmetric peaks for the longitudinal relaxation rate in the BPP plot for very large renewal rates.
\end{abstract}

\section{INTRODUCTION}

Disordered systems have found much interest in the last decades. Of particular interest are transport and relaxation processes in disordered materials. One aim of current research is to understand these time-dependent phenomena from the motional processes on atomic scales. Time-dependent processes can be investigated by many spectroscopic methods, such as nuclear magnetic resonance (NMR), electron spin resonance, quasielastic neutron scattering, Mössbauer effect, perturbed angular correlations, etc. To interpret the data it is necessary to know how the microscopic motional processes determine the measured signals in disordered systems, where averaging over many different events occurs. In this paper we develop a stochastic theory of spin relaxation in simple models for disordered systems. We consider relaxation of single spins; hence our theory is directly applicable to $\beta$ radiation-detected nuclear magnetic relaxation $(\beta-\mathrm{NMR})$ or muon spin relaxation ( $\mu \mathrm{SR}$ ) experiments, respectively, where polarized $\beta$-active nuclei or muons are used in extremely low concentrations as probe spins in condensed matter. ${ }^{1-3}$

The phenomenological description of spin relaxation was introduced by Bloch (see, e.g., Ref. 4 or 5 ) and the connection with microscopic fluctuation times was pointed out by Bloembergen, Purcell, and Pound ${ }^{6}$ (BPP). The theoretical basis of the BPP description was established by Wangsness and Bloch ${ }^{7}$ and Redfield ${ }^{8}$ (WBR). They related the longitudinal and transverse spin relaxation times $T_{1}$ and $T_{2}$, respectively, to correlation functions of the fluctuations that are acting on the spins. Essentially they used second-order time-dependent perturbation theory ${ }^{4,5}$ for the calculation of the expectation values of the spin components, whereby one presumes that the fluctuating spin-lattice interactions are small compared to the static interactions, which are due to the external field. One main assumption made in the derivations is that the correlation times of the fluctuations be short compared to all other relevant time scales. The BPP theory has been extremely successful for liquids and ordered solids, but the experimental results for defective and glassy solids do not fit into the frame provided by this theory. In this situation, many authors tried to modify the correlation functions of the WBR theory in ways that seem to be appropriate for disordered materials. However, this procedure is generally questionable, the reason being that in systems with static disorder the basic assumption of short correlation times is likely to be violated.

At low temperatures, disordered systems such as glasses are frozen in configurations with structural disorder. Different, isolated probe spins "feel" quite different local environments. A spin that is trapped in a local environment cannot, or only very rarely, make a transition to a completely different environment. Consequently, the correlation times for probing different field fluctuations become large, or even infinite. The procedure of using suitable correlation functions for disordered systems in evaluating the WBR expressions for $T_{1}^{-1}$ and $T_{2}^{-1}$ amounts to performing the homogeneous average $\left\{T_{1}^{-1}\right\}$ or $\left\{T_{2}^{-1}\right\}$ of the longitudinal or transverse relaxation rates. Here the braces designate the configurational average over different realizations of the disorder.

Several researchers realized that one should instead evaluate inhomogeneous averages in systems where the probe spins are frozen in different local configurations. ${ }^{9-13}$ Specifically they calculated the inhomogeneous average $\left\{\exp \left(-t / T_{1,2}\right)\right\}$ where the disorder is expressed by a distribution of $T_{1,2}$ values or, equivalently, by a distribution of correlation times. We agree fully with the basic idea of this approach, but we emphasize that restrictive assumptions have been made in the previous 
work. The essential assumption is that the local motion of the probe spin is so rapid that it leads to exponential relaxation, $\exp \left(-t / T_{1,2}\right)$. In disordered systems, the local motions may occur at quite different time scales and be even frozen at very low temperatures. In this paper we propose to consider the individual spin motions on a more microscopic scale. Instead of assuming exponential relaxation, we consider simple models for the motion of the probe spin in local environments. We average the resulting polarization decay over the disorder, which is expressed by distributions of the local rotation frequencies and hopping rates. Our approach might be considered as an extension of the classic stochastic theory of spin relaxation, which was originally developed by Anderson, ${ }^{14}$ Kubo and Tomita, ${ }^{15}$ and Kubo and Toyabe, ${ }^{16}$ to models of disordered systems. For instance, one well-known model for transverse spin relaxation is the one where a spin makes transitions with variable transition rates between two rotation frequencies. ${ }^{15}$ The three-dimensional extension of this model will be the basis for our averaging procedures. The models are rather simple; hence they can be treated more or less explicitly.

The relation of our work to the stochastic theory of spin relaxation by the authors of Refs. 14-16 has already been indicated. Spin relaxation where the Markov approximation of short correlation times is avoided has also been treated by other methods. In the work of Celio ${ }^{17} \mathrm{a}$ microscopic solution of the Schrödinger equation for the motion of a probe spin that interacts with the host spins is made by Trotter formula techniques. Stoeckmann ${ }^{18}$ developed a stochastic theory of spin relaxation where the dipolar interactions between the probe and the host spins are explicitly taken into account. In his work the motion of the probe spin in a lattice is treated by the effective-medium approximation and expressions that are appropriate for slow and fast motion are obtained. Diezemann and Schirmacher ${ }^{19}$ studied spin relaxation in high external fields by memory function techniques. The perturbation-theory approach of Ref. 19 is restricted to relatively strong external magnetic fields; hence it is not adequate for the experimental techniques we have in mind. Namely, in $\beta$-NMR and $\mu \mathrm{SR}$ the polarization of the probes does not depend on a Boltzmann factor as in conventional NMR and low magnetic fields can be applied without reduction in sensitivity. All papers mentioned do not include the characteristic dynamics of systems with frozen disorder that was described above. However, these papers might well serve as a basis for an extension of the present work.

The model that will be introduced in the subsequent section is a very simple two-site model. We find it worthwhile to relate the results for the model considered here as far as possible to concrete experimental realizations, which have been studied by $\mu \mathrm{SR}$ or $\beta$-NMR. Some remarks concerning the simplifications of the model are in order. We consider mainly the motion of single probe spins in static environments, and the interaction of the spin is assumed to be with the local environment. For example, in the lithium oxide glasses which are investigated by $\beta$-NMR (Ref. 2) there exists an approximately static structure which contains the mobile Li atoms. The
${ }^{8} \mathrm{Li}$ nuclei which serve as probes are dilute; hence their mutual interactions can be neglected. There is additional relaxation by the (unlike-spin) interaction of the ${ }^{8} \mathrm{Li}$ spins with the majority ${ }^{7} \mathrm{Li}$ spins. It amounts to a modification of the correlation time of the ${ }^{8} \mathrm{Li}$ spins, in analogy to the pure Li system. ${ }^{20}$ Our models are so crude that we disregard all these details; we work with an effective correlation time or inverse hopping rate which is considered as a parameter.

In the next section we investigate the two-frequency model for spin relaxation and we describe the various averaging procedures to be performed. In the third section we study the spin relaxation for the random twofrequency model on an intermediate time scale and compare the results with recent $\mu \mathrm{SR}$ experiments. The fourth section is devoted to an analysis of the long-time spin relaxation for the random two-frequency model. We introduce an effective longitudinal spin relaxation rate and examine whether its behavior as a function of the hopping rate can explain the features that were observed in glassy systems. In Sec. V we extend the random twofrequency model by admitting the possibility of temporal changes of the environment. Also transition processes of the probe spins between different environments may be incorporated in this way. The results of the stochastic modeling are not entirely satisfactory, as will be discussed in Sec. VI.

\section{RANDOM TWO-FREQUENCY MODELS}

The starting point of the stochastic theory of spin relaxation is the equation of motion for individual, classical spins $\vec{S}$,

$$
\frac{d}{d t} \vec{S}(t)=\vec{\omega}(t) \times \vec{S}(t) \equiv \underline{\underline{\Omega}}(t) \cdot \vec{S}(t)
$$

The spins are assumed to have unit lengths $|\vec{S}|=1$ and $\vec{\omega}(t)$ is the rotation frequency corresponding to the magnetic field experienced by the spin at time $t$. We will use the terms "rotation frequency" and "magnetic field" interchangeably. The rotation frequency $\vec{\omega}(t)$ may be decomposed into an external static field $\vec{\omega}_{0}$ and a local, fluctuating part $\vec{\omega}^{\prime}(t)$. The spin rotation may be alternatively described by the corresponding antisymmetric matrix $\underline{\Omega}(t)$. We assume complete polarization of the ensemble of spins at time $t=0$, expressed by the initial condition $\vec{S}(t=0)=\overrightarrow{S_{0}}$. The solution of Eq. (1) is trivial for fixed rotation frequency $\vec{\omega}$,

$$
\vec{S}(t)=\exp (\underline{\underline{\Omega}} t) \cdot \overrightarrow{S_{0}}
$$

\section{A. Two-frequency model}

The basis of the derivations in this paper is the rather simple "two-frequency" (TF) model. In this model which was introduced previously ${ }^{21}$ two sites with different magnetic fields $\vec{\omega}_{1}$ and $\vec{\omega}_{2}$ are considered and the spin jumps 
between the two frequencies according to a Poisson process with the hopping rate $\gamma=1 / \tau$. A pictorial representation of this process is given in Fig. 1. The quantities $\vec{\omega}_{1}$ and $\vec{\omega}_{2}$ may have arbitrary orientations with respect to $\overrightarrow{S_{0}}$, and the corresponding matrices $\underline{\Omega}_{1}, \underline{\Omega}_{2}$ do not commute. Because of this noncommutativity the problem is more difficult to treat than the textbook case of the transverse spin rotation. ${ }^{5}$ Nonetheless, the case of transitions between two arbitrarily oriented rotation frequencies can be solved analytically, ${ }^{21}$ as will be sketched below.

To obtain the average polarization $\vec{P} \equiv\langle\vec{S}(t)\rangle$ of an ensemble of spins, which are subjected to this hopping process in a single TF system, it is advantageous to make a decomposition of the polarization into the contributions of different numbers of state changes, ${ }^{22}$

$$
\vec{P}(t)=\sum_{l=0}^{\infty} \vec{P}_{l}(t)
$$

The quantity $\vec{P}_{l}(t)$ contains the contributions of all spins of the ensemble that have performed $l$ jumps until time $t$. It is a convolution of $l+1$ rotations $\exp (\underline{\underline{\Omega}} \Delta t)$, combined with the probabilities of performing a transition after the time interval $\Delta t$, which is given by $\gamma \exp (-\gamma \Delta t) .(\Delta t$ denotes arbitrary time intervals whose sum is the time $t$.) The result of the resummation of the series (3) in the Laplace domain is

$$
\tilde{\vec{P}}(s)=\frac{1}{2}\left[\left(\underline{\underline{D}}_{2}+\gamma \underline{\underline{1}}\right) \frac{1}{\underline{\underline{D}}_{1} \underline{\underline{D}}_{2}-\gamma^{2} \underline{\underline{1}}}+\left(\underline{\underline{D}}_{1}+\gamma \underline{\underline{1}}\right) \frac{1}{\underline{\underline{D}}_{2} \underline{\underline{D}}_{1}-\gamma^{2} \underline{\underline{1}}}\right] \cdot \overrightarrow{S_{0}},
$$

where the definition $\underline{\underline{D}}_{1,2} \equiv(s+\gamma) \underline{\underline{1}}_{-} \underline{\underline{\Omega}}_{1,2}$ has been introduced. Also the operators $\underline{\underline{D}}_{1}$ and $\underline{\underline{D}}_{2}$ do not commute.

Before discussing further the analytical solution Eq. (4) we point out the main features of the spin dynamics according to Eq. (4) in the two limiting cases of slow and rapid frequency chances compared to the rotation frequencies. In the former case it is easy to see from the series Eq. (3) that the spins will precess with their respective starting frequencies, nearly independently of the other frequency. In contrast, in the case of rapid transitions $\left(\gamma \gg\left|\vec{\omega}_{1}\right|,\left|\vec{\omega}_{2}\right|\right)$ we find rotation of the spins that corresponds to static spins which perform Larmor precession with the rotation frequency $\left(\vec{\omega}_{1}+\vec{\omega}_{2}\right) / 2$, the rotation being weakly damped. This feature can be made plausible by appeal to the Trotter formula; see Ref. 21. That is, for rapid state changes we may disregard the noncommutability of $\underline{\underline{\Omega}}_{1}, \underline{\Omega}_{2}$ or of $\underline{\underline{D}}_{1}, \underline{\underline{D}}_{2}$, respectively. Then Eq. (4) reduces to

$$
\tilde{\vec{P}}(s) \approx\left([s+2 \gamma] \underline{\underline{1}}-\frac{1}{2}\left(\underline{\underline{\Omega}}_{1}+\underline{\underline{\Omega}}_{2}\right)\right)\left(\left(s^{2}+2 \gamma s\right) \underline{\underline{1}}-(s+\gamma)\left(\underline{\underline{\Omega}}_{1}+\underline{\underline{\Omega}}_{2}\right)+\underline{\underline{\Omega}}_{1} \cdot \underline{\underline{\Omega}}_{2}\right)^{-1} \cdot \overrightarrow{S_{0}} .
$$

For large $\gamma$, the rotation is damped corresponding to the usual motional-narrowing process.

In Ref. 21 two special zero-field cases $\vec{\omega}_{0}=0$ were considered, namely, $\vec{\omega}_{1}^{\prime}=\omega_{x} \vec{e}_{x}, \vec{\omega}_{2}^{\prime}=\omega_{z} \overrightarrow{e_{z}}$, and $\vec{\omega}_{1}^{\prime}=$ $\omega_{x} \vec{e}_{x}, \vec{\omega}_{2}^{\prime}=\omega_{y} \vec{e}_{y}$, respectively. The initial condition was $\vec{S}_{0}=\vec{e}_{z}$ and the longitudinal polarization $P_{z}(t)$ was derived. We use the same initial conditions and restrict ourselves to the longitudinal polarization also in the general case. The derivation given below can be extended to the other components of the polarization. Since the rotation matrices are three dimensional, the denominators of Eq. (4) are polynomials of order 6 in the Laplace variable $s$, and the $z$ component of Eq. (4) can be represented in the form

$$
\tilde{P}_{z}(s)=\frac{1}{2} \frac{a(s)}{b(s)}=\frac{1}{2}\left[\sum_{i=0}^{5} a_{i} s^{i}\right]\left[\sum_{j=0}^{6} b_{j} s^{j}\right]^{-1}
$$

The coefficients $a_{i}$ and $b_{j}$ were determined from Eq. (4) by algebraic formula manipulation with REDUCE. They are explicitly given in the Appendix.

In principle, the solution (6) can be transformed into the time domain using the Heaviside expansion theorem. ${ }^{23}$ One obtains

$$
P_{z}(t)=\frac{1}{2} \mathcal{L}^{-1}\left\{\frac{a(s)}{b(s)}\right\}=\sum_{n=1}^{6} \frac{a\left(s_{n}\right)}{b^{\prime}\left(s_{n}\right)} \exp \left(s_{n} t\right) .
$$

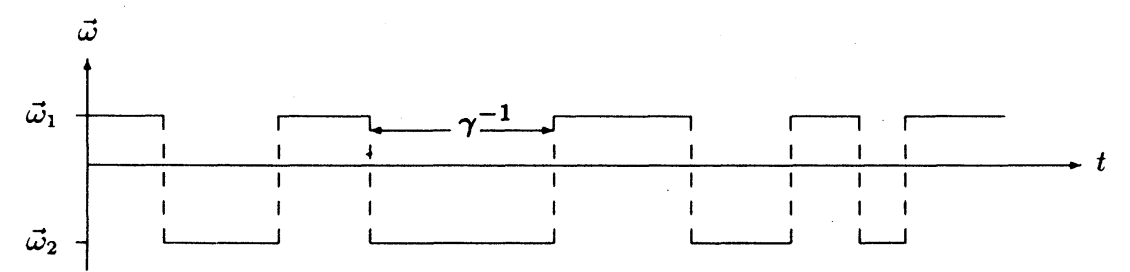

FIG. 1. Schematic representation of the jump process as a function of time between two sites which are characterized by the rotation frequencies $\vec{\omega}_{1}$ and $\vec{\omega}_{2}$. The mean time between two frequency changes is $1 / \gamma$. 
In this equation $s_{n}(n=1, \ldots, 6)$ denote the different nondegenerate roots of the polynomial $b(s)$. The case of degenerate roots requires special attention. ${ }^{24}$ The quantity $P_{z}(t)$ represents then the $z$ component of the spin polarization, averaged over the hopping processes of the spin.

Since the polynomial $b(s)$ in Eq. (6) is of order 6 , it is not possible to calculate its roots in general. Nevertheless, by considering the limiting case of rapid fluctuations, we are able to determine approximately the roots of $b(s)$ by expanding the denominator of Eq. (6) up to second order in $\left|\vec{\omega}_{1}\right| / \gamma$ or $\left|\vec{\omega}_{2}\right| / \gamma$, respectively. This procedure which is described in detail in Ref. 24 leads then in lowest order to the longitudinal polarization

$$
P_{z}(t)=\frac{\omega_{\mathrm{res}, z}^{2}}{\vec{\omega}_{\mathrm{res}}^{2}} \exp \left[-\frac{t}{\gamma} f_{1}\left(\vec{\omega}_{1}, \vec{\omega}_{2}\right)\right]+\left[\cos \left(\left|\vec{\omega}_{\mathrm{res}}\right| t\right) \frac{\vec{\omega}_{\mathrm{res}}^{2}-\omega_{\mathrm{res}, z}^{2}}{\vec{\omega}_{\mathrm{res}}^{2}}+\sin \left(\left|\vec{\omega}_{\mathrm{res}}\right| t\right) \cdot \mathcal{O}\left(\frac{1}{\gamma}\right)\right] \exp \left\{-\mathcal{O}\left(\frac{1}{\gamma}\right)\right\}
$$

with the function $f_{1}\left(\vec{\omega}_{1}, \vec{\omega}_{2}\right)=\left[\vec{\omega}_{1}^{2} \vec{\omega}_{2}^{2}-\left(\vec{\omega}_{1} \cdot \vec{\omega}_{2}\right)^{2}\right] /\left(8 \vec{\omega}_{\text {res }}^{2}\right)$ and the averaged frequency $\vec{\omega}_{\text {res }}=\left(\vec{\omega}_{1}+\vec{\omega}_{2}\right) / 2$. Evidently this solution corresponds to the polarization decay that would follow from Eq. (5).

In the derivation of Eqs. (7) and (8) it was assumed that the roots of the denominator of Eq. (6) are not degenerate. They are degenerate, for example, if the condition $\left|\vec{\omega}_{\text {res }}\right|=0$ holds. This condition means $\vec{\omega}_{1}=-\vec{\omega}_{2}$, and we assume $\left|\vec{\omega}_{1,2}\right| \neq 0$ (otherwise the solution is trivial). Under this condition, the poles of Eq. (6) can be determined, and one obtains the following polarization decay which depends on the sign of the root $\sqrt{\gamma^{2}-\vec{\omega}_{1}^{2}}$ :

$$
\begin{aligned}
& \text { (a) } \gamma^{2}>\vec{\omega}_{1}^{2}: P_{z}(t)=\left\{\frac{\gamma}{\sqrt{\gamma^{2}-\vec{\omega}_{1}^{2}}} \sinh \left[\sqrt{\gamma^{2}-\vec{\omega}_{1}^{2}} t\right]+\cosh \left[\sqrt{\gamma^{2}-\vec{\omega}_{1}^{2}} t\right]\right\} \frac{\vec{\omega}_{1}^{2}-\omega_{1 z}^{2}}{\vec{\omega}_{1}^{2}} \exp (-\gamma t)+\frac{\omega_{1 z}^{2}}{\vec{\omega}_{1}^{2}} ; \\
& \text { (b) } \gamma^{2}=\vec{\omega}_{1}^{2}: P_{z}(t)=\frac{\omega_{1 z}^{2}}{\vec{\omega}_{1}^{2}}+\frac{\vec{\omega}_{1}^{2}-\omega_{1 z}^{2}}{\vec{\omega}_{1}^{2}} \exp (-\gamma t)\{\gamma t+1\} ; \\
& \text { (c) } \gamma^{2}<\vec{\omega}_{1}^{2}: P_{z}(t)=\left\{\frac{\gamma}{\sqrt{\vec{\omega}_{1}^{2}-\gamma^{2}}} \sin \left[\sqrt{\vec{\omega}_{1}^{2}-\gamma^{2}} t\right]+\cos \left[\sqrt{\vec{\omega}_{1}^{2}-\gamma^{2}} t\right]\right\} \frac{\vec{\omega}_{1}^{2}-\omega_{1 z}^{2}}{\vec{\omega}_{1}^{2}} \exp (-\gamma t)+\frac{\omega_{1 z}^{2}}{\vec{\omega}_{1}^{2}} .
\end{aligned}
$$

The case $\vec{\omega}_{1}=-\vec{\omega}_{2}$ is somewhat more general than the transverse case with zero mean frequency, since $\vec{\omega}_{1}$ may form an arbitrary angle with $\vec{S}_{0}$. We refer to Ref. 24 for further details about the treatment of Eq. (6) and the resulting polarization decay.

\section{B. Configurational averages: Averages over the hopping rates}

The disorder of the systems under consideration is taken into account by averaging the polarization decay $P_{z}(t)$ of a single subsystem over the ensemble of subsystems, assuming that the rotation frequencies $\vec{\omega}_{1}$, $\vec{\omega}_{2}$ are random variables. The problem is to specify appropriate distributions of the rotation frequencies. The most commonly used distribution is the Gaussian one. If we assume that the components of the frequencies $\vec{\omega}_{1}, \vec{\omega}_{2}$ are distributed around $\vec{\omega}_{0}$ with the variance $\sigma$, we have to evaluate

$$
\left\{P_{z}(t)\right\}_{\vec{\omega}_{1}, \vec{\omega}_{2}}=\frac{1}{\left(2 \pi \sigma^{2}\right)^{3}} \int_{-\infty}^{\infty} d \vec{\omega}_{1} d \vec{\omega}_{2} P_{z}(t) \exp \left[-\frac{\left(\vec{\omega}_{1}-\vec{\omega}_{0}\right)^{2}+\left(\vec{\omega}_{2}-\vec{\omega}_{0}\right)^{2}}{2 \sigma^{2}}\right]
$$

Either the inverse Laplace transform of Eq. (6) or the result of Eq. (7) for $P_{z}(t)$ has to be used in the integral. The average over the quenched disorder is generally indicated by the braces \{\} .

The ansatz of a Gaussian distribution of rotation frequencies can also reflect the frequency distributions in crystalline systems that are caused by dipolar interactions between nuclear spins. ${ }^{25}$ As discussed in the Introduction, we argue that the characteristic spin relaxation of glassy systems is due to structural disorder. The Gaussian distribution used in Eq. (10) may not be appropriate to represent structural disorder. Therefore we examined also two other distributions, namely, the rectangular distribution where

$$
\left\{P_{z}(t)\right\}_{\vec{\omega}_{1}, \vec{\omega}_{2}}=\frac{1}{\left(2 \sqrt{3} \sigma_{r}\right)^{3}} \int_{\omega_{0}-\sqrt{3} \sigma_{r}}^{\omega_{0}+\sqrt{3} \sigma_{r}} d \vec{\omega}_{1} d \vec{\omega}_{2} P_{z}(t)
$$

and the symmetric exponential distribution where

$$
\left\{P_{z}(t)\right\}_{\vec{\omega}_{1}, \vec{\omega}_{2}}=\frac{1}{\left(\sqrt{2} \sigma_{e}\right)^{3}} \int_{-\infty}^{\infty} d \vec{\omega}_{1} d \vec{\omega}_{2} P_{z}(t) \exp \left[-\frac{\sqrt{2}\left(\left|\vec{\omega}_{1}-\vec{\omega}_{0}\right|+\left|\vec{\omega}_{2}-\vec{\omega}_{0}\right|\right)}{\sigma_{e}}\right]
$$


We do not expect significant differences between the Gaussian and the rectangular distributions, but the exponential distribution is different in that it contains small and large frequency deviations from the value $\vec{\omega}_{0}$ with a completely different weighting than in the Gaussian distribution.

Further, we also investigate the influence of distributions of the hopping rate $\gamma$; i.e., we assume that this rate is a random variable, too. The physical picture behind this assumption is that the jump rates of spins in subsystems of the disordered medium may be very different. Explicitly, we studied rectangular distributions of the rate $\gamma$, expressed by

$$
\left\{P_{z}(t)\right\}_{\vec{\omega}_{1}, \vec{\omega}_{2}, \gamma}=\frac{1}{2 \sqrt{3} \sigma_{\gamma r}} \int_{0}^{2 \sqrt{3} \sigma_{\gamma r}} d \gamma\left\{P_{z}(t)\right\}_{\vec{\omega}_{1}, \vec{\omega}_{2}}
$$

and exponential distributions, which lead to

$$
\left\{P_{z}(t)\right\}_{\vec{\omega}_{1}, \vec{\omega}_{2}, \gamma}=\frac{1}{\sigma_{\gamma e}} \int_{0}^{\infty} d \gamma \exp \left(-\frac{\gamma}{\sigma_{\gamma e}}\right)\left\{P_{z}(t)\right\}_{\vec{\omega}_{1}, \vec{\omega}_{2}} .
$$

The average polarization decay $\left\{P_{z}(t)\right\}_{\vec{\omega}_{1}, \vec{\omega}_{2}}$ to be used in the integrals is determined by the Gaussian average (10). Since we want to examine the influence of lower jump rates on the time development of the relaxation, the integration starts at the lower limit $\gamma=0$. In particular, the exponential distribution Eq. (14) includes a wide span of different jump rates.

In most cases, the averages expressed by Eqs. (10)(14) cannot be calculated analytically. Hence we have to resort to numerical procedures to perform the required integrations. It is advisable to use different quadrature rules for the integrations which are adapted to the respective frequency or hopping-rate distributions. Unfortunately, the integrand $P_{z}(t)$ does not behave like a polynomial for which the quadrature rules may become exact from a certain order on. Since generally sixfold integrations are involved, the choice of the order $n$ of the quadrature rule is of concern, where a compromise has to be made between accuracy of the results for $\left\{P_{z}(t)\right\}$ and required CPU time. The inaccuracy of the integration becomes large when the value of the rotation frequency is approximately equal to the hopping rate and it increases with increasing time coordinate $t$ (see Ref. 24 for details).

\section{LONGITUDINAL SPIN RELAXATION IN THE RANDOM TF MODEL AT INTERMEDIATE TIME SCALES}

This section gives results on the longitudinal spin relaxation for the random two-frequency model at intermediate time scales. It is the strength of stochastic models of spin relaxation that they yield information on the line shape, or, in the time domain, on the transients of the polarization decay. We will present results for the random TF (RTF) model, where the rotation frequencies $\vec{\omega}_{1}, \vec{\omega}_{2}$ are averaged over Gaussian distributions in threedimensional spaces; cf. Eq. (10).

\section{A. Results for the RTF model}

We average the polarization decay $P_{z}(t)$ for the basic TF model over a Gaussian frequency distribution of unit width $\sigma$ and external zero field, for various values of the hopping rate $\gamma$. For this purpose, the result Eq. (6) in the Laplace domain is transformed numerically to the time domain by the fast inversion routine described in Ref. 26 . The disorder average is then performed numerically, as described in Sec. II B. The resulting spin relaxation is shown in Fig. 2. For $\gamma \rightarrow 0$ the longitudinal polarization is characterized by the static Kubo-Toyabe function ${ }^{16}$

$$
\begin{aligned}
\left\{P_{z}(t)\right\}_{\vec{\omega}_{1}, \vec{\omega}_{2}} & =\frac{1}{3}+\frac{2}{3}\left(1-\sigma^{2} t^{2}\right) \exp \left(-\frac{1}{2} \sigma^{2} t^{2}\right) \\
& \equiv g_{\mathrm{KT}}\left(t ; \sigma, \omega_{0}=0\right) .
\end{aligned}
$$

The minimum of the polarization decay is located at $t_{\min }=\sqrt{3} / \sigma$. The increase of the jump rate $\gamma$ leads to a shift of the minimum to larger times. In addition, the recovery of the polarization to the asymptotic value $1 / 3$ is no longer observed. Whereas these features resemble qualitatively the results of the strong-collision model of Hayano et al. $^{27}$ (see the inset in Fig. 2), the polarization transients of both models behave completely differently in the parameter region $\gamma \geq \sigma$. There, the longitudinal polarization of the RTF model increases again for $t>t_{\min }$ and furthermore, for $\gamma \rightarrow \infty$, the polarization approaches a limiting curve, namely, a static KuboToyabe function according to Eq. (15) where the parameter $\sigma$ is reduced by a factor of $\sqrt{2}$. Nevertheless, it should be noted that all polarization transients decay down to zero for larger time (a fact which cannot be made visible in Fig. 2) apart from the limiting cases $\gamma=0$ and $\gamma \rightarrow \infty$. This point will be further investigated in Sec. V.

The results for $\gamma \gg \sigma$ are in agreement with Eq. (5) and the qualitative discussion in Sec. II A. Namely, for large hopping rates, one has an effective spin precession around the resulting frequency $\left(\vec{\omega}_{1}+\vec{\omega}_{2}\right) / 2$ and the width of the corresponding distribution is reduced by a factor of $\sqrt{2}$. It is instructive to contrast this behavior with that of the strong-collision model, where at each transition a new frequency is selected. This leads to the disappearance of the minimum and the polarization decays monotonically for large $\gamma$; cf. the inset in Fig. 2.

Finally, also a longitudinal external field $\vec{\omega}_{0}=\omega_{0} \vec{e}_{z}$ can be considered. The Kubo-Toyabe function is well known for this case $;{ }^{27}$ the minimum is less pronounced for finite $\omega_{0}$. For this situation, the RTF model shows a behavior whose qualitative features correspond to the behavior for $\omega_{0}=0$. That is, for intermediate $\gamma$ the Kubo-Toyabe function is smoothed out, and for large $\gamma$ the minimum is again shifted to larger times by the factor $\sqrt{2}$. More details are given in Refs. 21, 24 . 


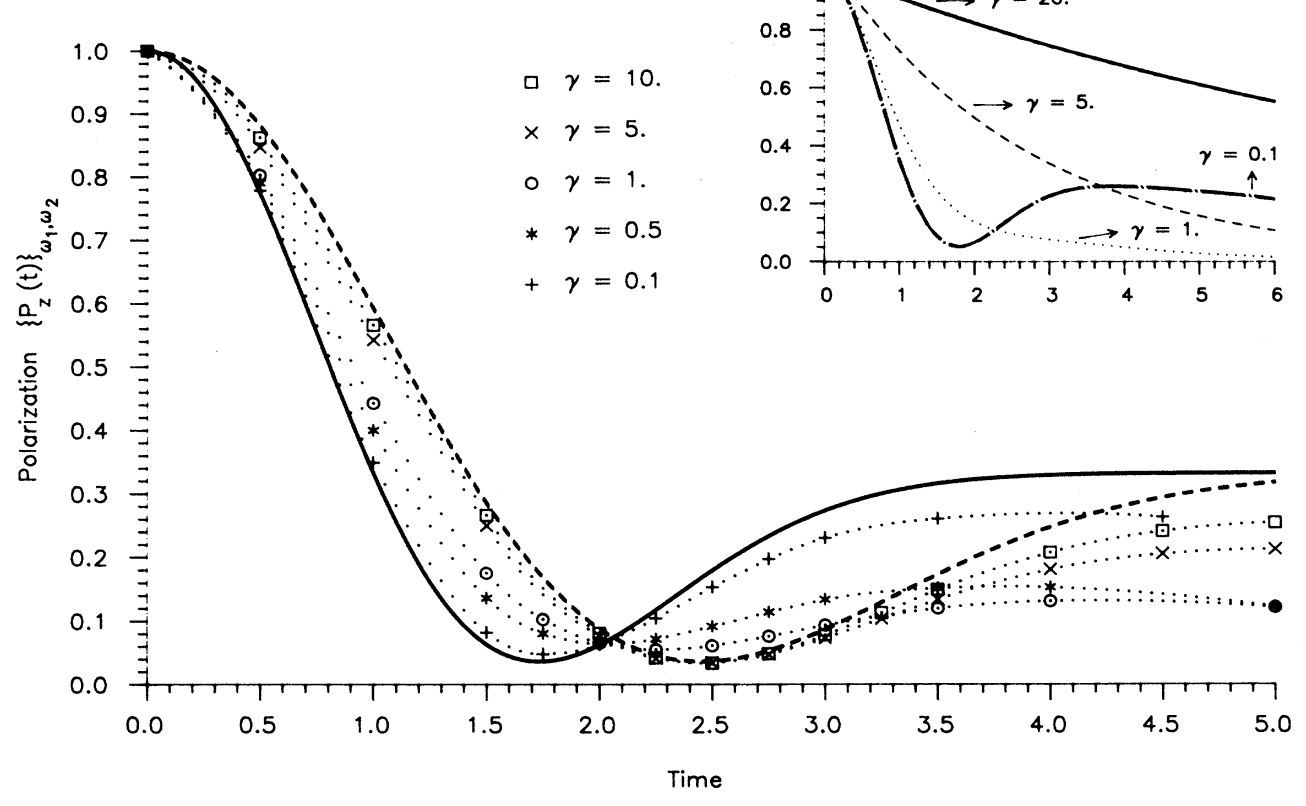

FIG. 2. Average polarization $\left\{P_{z}(t)\right\}_{\vec{\omega}_{1}, \vec{\omega}_{2}}$, Eq. (10), for fixed parameters $\sigma=1, \vec{\omega}_{0}=0$, and different values of the hopping rate $\gamma$. The rates are given as multiples of a reference rate whose inverse serves as the time unit. The solid (dashed) line represents the solution (15) for $\sigma=1(\sigma=1 / \sqrt{2})$, whereas the dotted lines serve as visual support. The inset shows the results of the "strong-collision" model of Hayano et al. (Ref. 27).

\section{B. Qualitative experimental confirmation of the RTF model}

The behavior of the spin relaxation on intermediate time scales that was analyzed in the last subsection can be observed in muon spin rotation ( $\mu \mathrm{SR})$ experiments. $^{3}$ The width parameter $\sigma$ of several substances fits to the inverse of the lifetime of the muons, such that the effects addressed in Sec. III A are in a suitable time window. Fell et $a l .^{28}$ studied the longitudinal muon spin relaxation in niobium doped with titanium for the zero-field case. Figure 3 shows the measured polarization transients at three different temperatures. At low temperatures $(T=13$
K) one recognizes Kubo-Toyabe behavior of the polarization decay. For increasing temperatures $(T=22 \mathrm{~K})$ a stronger polarization decay is observed and the KuboToyabe minimum no longer exists. A further increase of the temperature (up to $T=57 \mathrm{~K}$ ) leads to a recovery of the Kubo-Toyabe behavior, where the region of the minimum is shifted to longer times. In experimental paper ${ }^{28}$ the occurrence of minima in the polarization decay functions was interpreted as an indication of localization of the muons at the corresponding temperatures. The delocalization of the muons over four or six sites at the higher temperature of $57 \mathrm{~K}$ was inferred from the decay constants of the transverse polarization. An interpreta-

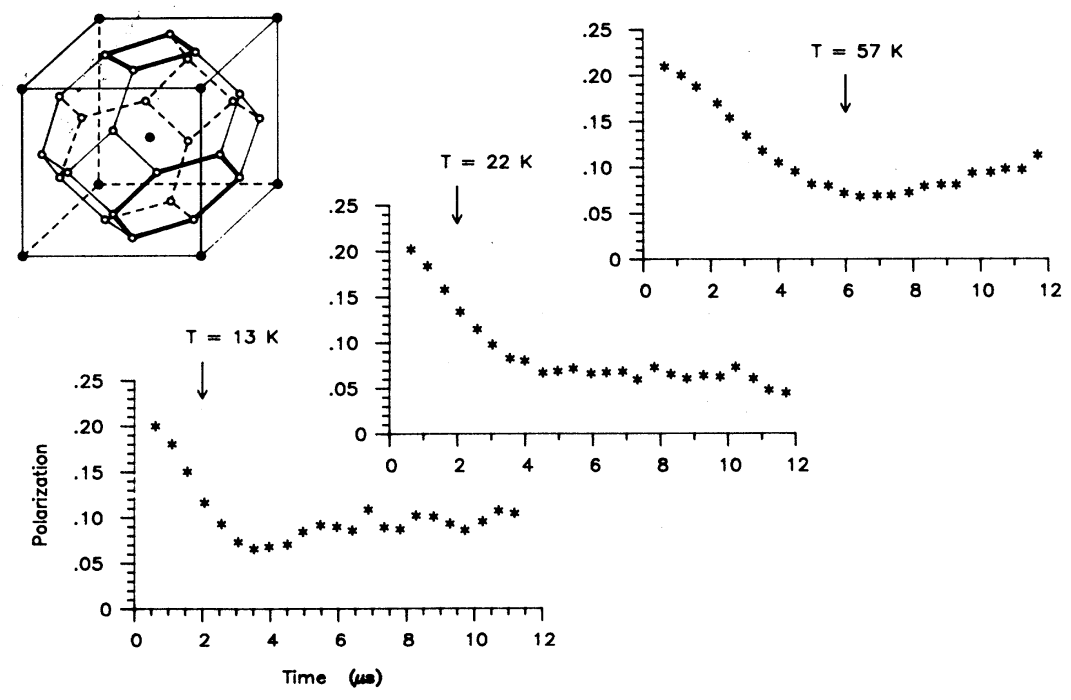

FIG. 3. Longitudinal polarization decay of muons in $\mathrm{Nb}_{0.99} \mathrm{Ti}_{0.01}$ for three values of the temperature from Ref. 28. The inset represents the unit cell of a body-centered lattice. The solid circles mark the $\mathrm{Nb}$ atoms or a $\mathrm{Ti}$ impurity at the center, whereas the small open circles represent the 24 interstitial tetrahedral sites adjacent to a Ti impurity. The thick bonds indicate the possible local diffusion over four or six sites, respectively. 
tion of the difference of the minimum positions in the longitudinal polarization decay at the two temperatures of $13 \mathrm{~K}$ and $57 \mathrm{~K}$ was not given.

The disappearance of the minimum and its reappearance at a shifted position with increasing temperature are readily explained by the following picture. At the lowest temperature, the muons are localized at the tetrahedral interstitial sites in the $\mathrm{Nb}$ lattice and exhibit KuboToyabe behavior in zero external field, which reflects the approximate Gaussian distribution of the dipolar magnetic fields due to the surrounding $\mathrm{Nb}$ nuclei. At the intermediate temperature the muon performs jumps; this leads to the disappearance of the Kubo-Toyabe behavior, as expected from the strong-collision model. At still higher temperatures, the muons will be trapped near the Ti impurities, but they can still perform local motion over several sites. Candidates for groups of sites over which local motion takes place are rings of four or six sites; see the inset in Fig. 3. Delocalization of light particles over several sites has been observed, e.g, for hydrogen in $\mathrm{Nb}$ with impurities. ${ }^{29}$ If the muons perform rapid local motion over several sites, they experience effectively the average of the local magnetic fields over the sites involved; this leads to a Kubo-Toyabe behavior analogous to the one derived for the RTF model. We emphasize that only two theories yield minima in the longitudinal polarization function as a function of time, the Kubo-Toyabe theory ${ }^{15}$ and the (more microscopic) theory of Celio. ${ }^{17}$ Our extension of the Kubo-Toyabe theory readily explains the occurrence of minima at different positions, depending on the magnitude of the transition rate.

There remains the task of a quantitative interpretation. While the RTF model predicts a shift of the maximum by the factor 1.414 , an apparent shift by a factor 1.85 can be taken from Fig. 3. One expects that the extension of the random two-frequency model to an $n$ frequency model yields a shift of the minimum position to $\sqrt{n} t_{\text {min }}$ for large transition rates, if all frequencies are uncorrelated. An analogous reduction of the width parameter appears in the inhomogeneous static polarization decay in the transverse case, if the muons are delocalized over several sites. ${ }^{30}$ Thus the extension of the arguments of the RTF model to four or six sites would give shift factors of 2 or 2.45 , respectively, if all frequencies were uncorrelated. The dipolar fields at the rings of four or six sites are evidently correlated, since these sites have common neighbor nuclei. Calculations for the average dipolar fields on rings of four and six sites were reported by Lankford et $a l .^{31}$ If we average their results over the field orientations, we obtain a reduction factor of 1.36 or 2.10 , respectively. Hence the observed value would suggest a delocalization of the muons over six sites. Of course, for a more detailed interpretation of the previous work, ${ }^{28}$ or of possible future investigations, random models with four or six sites including correlated Larmor frequencies should be explicitly treated. In Ref. 24 a model was introduced where correlations between the rotation frequencies at neighboring sites were taken into account. The results could be described by the introduction of an effective width which enters the Kubo-Toyabe function.

\section{RELAXATION RATES OF THE ASYMPTOTIC POLARIZATION DECAY}

\section{A. Determination of the asymptotic decay}

The stochastic theory of spin relaxation is apparently successful in describing longitudinal spin relaxation in disordered situations at the intermediate time scales that are used in $\mu \mathrm{SR}$ experiments. The question arises whether it is also possible to obtain useful results for the long-time behavior of the longitudinal spin relaxation, such as it is observed on the typical time scales of NMR or $\beta$-NMR experiments. Is it possible to resolve the problems that appeared when these experiments were analyzed in terms of the standard NMR theory? We will address this problem from an applied point of view by evaluating the longitudinal spin relaxation for the RTF model with typical experimental parameters.

The configurational average over disordered frequencies naturally leads to nonexponential polarization decay. Ideally one should compare theory and experiment over extended time scales, spanning many orders of magnitude. In practice, experiments often are restricted to rather small time windows; in addition the data are noisy. It is common practice to reduce the data by introducing relaxation rates, but this already can be done in different ways. A rough estimate is provided by the inverse time of decay of the signal to $e^{-1}$; a better procedure is to fit an exponential decay to the data within the time window. A popular procedure is to employ the Kohlrausch-WilliamsWatts (KWW) function (see, e.g., Refs. 32 and 33 for the use of this function in a different context)

$$
P_{z}(t) \sim \exp \left(-\left[T_{1}^{-1} t\right]^{\alpha}\right)
$$

In Ref. 13 the longitudinal relaxation time is defined by the time integral over the polarization decay function. We will use Eq. (16) in our work since it has been employed in many papers to parametrize the polarization decay observed experimentally in finite time windows. In the following, the calculation of the polarization decay of the investigated models is performed for parameter values which are typical for $\beta$-NMR measurements with the probe nucleus ${ }^{8} \mathrm{Li}^{34}$ In detail, we choose a Larmor frequency $\omega_{0} \sim 10^{6} \mathrm{~s}^{-1}$ and a variance of the distribution $\sigma \equiv 10^{4} \mathrm{~s}^{-1}$. Furthermore, the hopping rates are within the region $\gamma \leq 10^{10}-10^{12} \mathrm{~s}^{-1}$ and the investigations are restricted to the time window [0.01-0.1] s. For these parameter values we are clearly in the long-time limit which is determined by the condition $t \gg 1 / \gamma$ or $s \ll \gamma$ in Laplace space, respectively.

The expression (6) for the spin polarization of the TF model derived in Sec. II A has four complex conjugate and two real roots. ${ }^{35}$ The complex conjugate roots in the Laplace domain represent damped spin rotation in the time domain. These spin rotations lead to Gaussian decay of that part of the polarization when the average over different rotation frequencies is performed. This means that the contributions of the corresponding poles is negli- 
gible in the long-time limit. There remain two real roots of Eq. (6); only one of them is small and determines the long-time behavior.

For computational convenience we made an expansion of the numerator of Eq. (6) up to first order in $s$ and of the denominator up to second order,

$$
\underline{\underline{\tilde{P}}}_{z z}^{\gamma}(s) \approx \frac{1}{2} \frac{a_{0}+a_{1} s}{b_{0}+b_{1} s+b_{2} s^{2}},
$$

with the coefficients from Eq. (A1). This expression has two real roots of which the smaller one coincides practically with the one of the complete expression. We convinced ourselves that the use of Eq. (17) leads to deviations from $P_{z}(t)$ that would follow from Eq. (6) of at most $3 \%$. On the other hand a considerable reduction of computing time is achieved, since now an analytic form can be used when performing averages. Namely, Eq. (17) reads in the time domain (with the initial condition $\overrightarrow{S_{0}}=\overrightarrow{e_{z}}$ )

$$
\begin{aligned}
P_{z}(t)= & \frac{1}{2 b_{2}}\left\{\frac{a_{0}}{s_{1}-s_{2}}\left[\exp \left(s_{1} t\right)-\exp \left(s_{2} t\right)\right]\right. \\
& \left.+\frac{a_{1}}{s_{1}-s_{2}}\left[s_{1} \exp \left(s_{1} t\right)-s_{2} \exp \left(s_{2} t\right)\right]\right\} .
\end{aligned}
$$

The quantities $s_{1,2}$ denote the roots of the denominator,

$$
s_{1,2}=\frac{1}{2 b_{2}}\left[-b_{1} \pm \sqrt{b_{1}^{2}-4 b_{0} b_{2}}\right]
$$

whereby the coefficients are given by Eq. (A1). In the special case of $b_{2}=0$ where the roots are manifold degenerate Eq. (18) reduces to $P_{z}(t) \equiv 1, \forall t$.

\section{B. Results for the RTF model}

We calculate numerically the long-time behavior of the polarization decay using Eq. (18) and performing averages over Gaussian distributions of the rotation frequencies; cf. Eq. (10). By fitting each transient with the KWW function (16) we obtain the quantities $T_{1}^{-1}$ and $\alpha$, respectively, which are plotted in Figs. 4(a) and 4(b) versus the hopping rates $\gamma$ for four different Larmor frequencies. The typical BPP shape of this double-logarithmic plot illustrates generally that the applied procedure and the discussed model yield reasonable results; i.e., the obtained values of the relaxation rate are at least qualita-

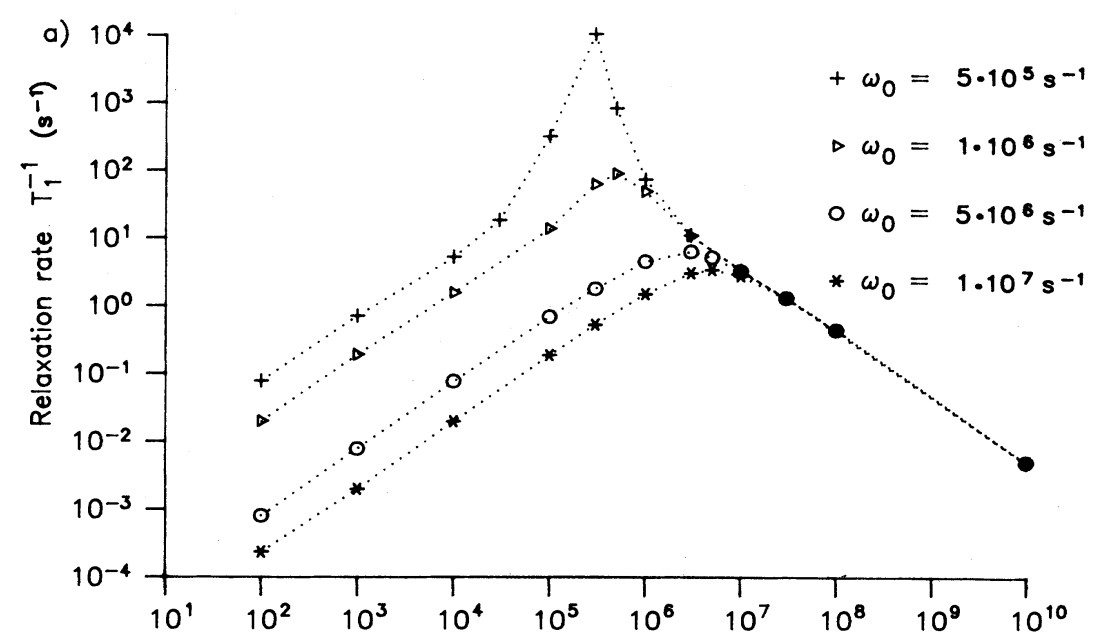

FIG. 4. (a) Plot of the relaxation rate $T_{1}^{-1}$ vs the hopping rate $\gamma$ for different external Larmor frequencies $\omega_{0}$, for the case of a Gaussian frequency distribution with $\sigma=1 \mathrm{~s}^{-1}$. (b) Plot of the exponent $\alpha$ of

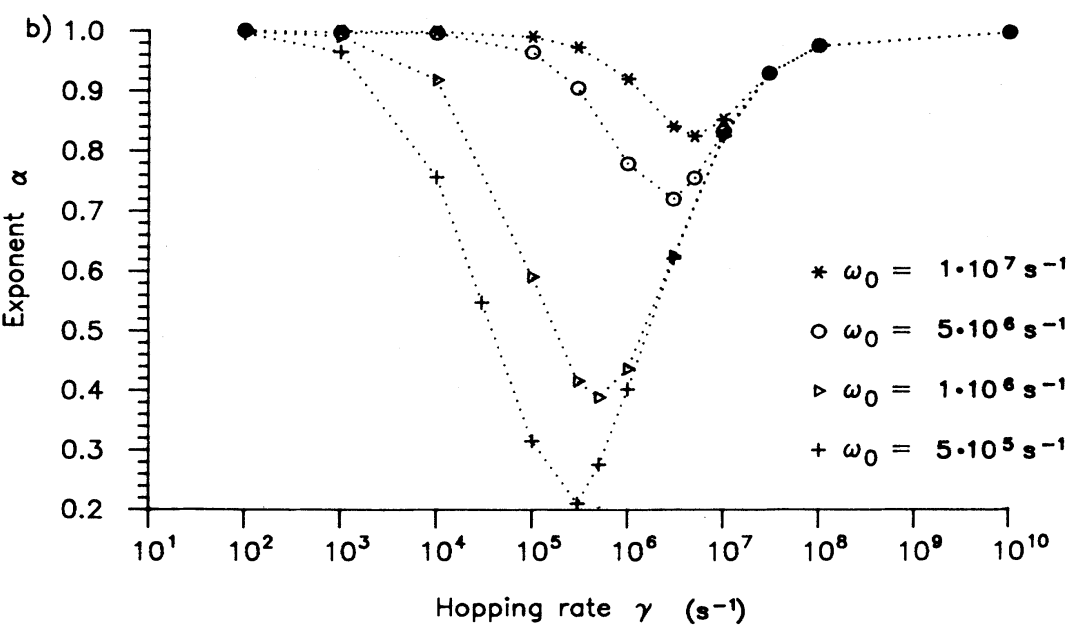
the stretched-exponential fit function vs the hopping rate. 
tively comparable to the rates measured, e.g., in $\mathrm{LiCl} \cdot n$ $\mathrm{D}_{2} \mathrm{O}$ with the $\beta$-NMR method. ${ }^{34}$

We now examine the quantitative features of Fig. 4.

(i) The relaxation rate $T_{1}^{-1}$ shows quite similar behavior as a function of the hopping rate as in standard NMR theory in that it has a symmetric maximum with the same slopes on both sides.

(ii) The relaxation rates are independent of the Larmor frequency in the regime of large hopping rates ("hightemperature side"), whereas the rates show such a dependence for small hopping rates with the behavior (for $\gamma=10^{3} \mathrm{~s}^{-1}$ )

$$
T_{1}^{-1} \sim \omega_{0}^{-1.96}
$$

This behavior is similar to the BPP theory which gives $T_{1}^{-1} \sim \omega_{0}^{-2}$.

(iii) Obviously, the curves in Fig. 4(a) show a significant hump for low Larmor frequencies which has not yet been observed in experiment. ${ }^{36} \mathrm{Up}$ to now it is not clear whether this feature describes a real physical property or whether it reflects an artifact of the model; see also the discussion in Sec. VD. A greater accuracy of the integration procedures does not change this hump significantly. ${ }^{24}$

(iv) Finally, a Kohlrausch exponent $\alpha$ is observed which is significantly different from one for large ranges of the parameters. The exponent $\alpha$ depends on both the magnetic field and the hopping rate as is shown in Fig. 4(b). Generally $\alpha$ decreases down to a minimum value at $\gamma \simeq \omega_{0}$, whereas it reaches the value one in the limiting cases of slow and rapid fluctuations. Analogous to the behavior of the relaxation rate, an increase of the Larmor frequency leads to a shift of the minimum regime to larger hopping rates $\gamma$; simultaneously the deviation from the simple-exponential decay $(\alpha=1)$ decreases.

A dependence of the Kohlrausch exponent $\alpha$ on magnetic field and temperature has experimentally been verified by measurements of the nuclear spin-lattice relaxation in several glasses. For example, Schnauss et $a l .{ }^{11,12}$ observed $\alpha<1$ in orthoterphenyl and glycerol around and below the glass transition temperature by ${ }^{2} \mathrm{H}-\mathrm{NMR}$ spin-lattice experiments. In analogous experiments on toluol ${ }^{37}$ it was found that $\alpha$ depends on $\omega_{0}$ in a way which qualitatively agrees with that observed on the low-temperature side of the dip in Fig. 4. There is also some indication for a field.. and temperaturedependent Kohlrausch exponent in the inorganic glass $\mathrm{Li}_{2} \mathrm{O} \cdot \mathrm{Al}_{2} \mathrm{O}_{3} \cdot 4 \mathrm{SiO}_{2} \cdot{ }^{38}$

To summarize the results up to this point, we may say that the RTF model with a Gaussian distribution of the rotation frequencies seems to be supported by experiments as far as the results for the Kohlrausch exponent are concerned. On the other hand this model cannot explain the significant deviations from the BPP behavior that were observed for the NMR relaxation rates of disordered materials.

It was already pointed out in Sec. IIB that the Gaussian frequency distribution may not describe the dynamical behavior of disordered systems in an adequate way. Therefore, we investigated alternatively the average of the polarization decay in each subsystem over the rectangular frequency distribution (11) and the symmetrical exponential distribution (12), respectively. However, the plots of the relaxation rate $T_{1}^{-1}$ and of the exponent $\alpha$ exhibit qualitatively the same results as for the Gaussian frequency distribution. The different frequency weights in the various distributions cause only slight modifications of the shape and of the extreme values. Evidently the symmetric exponential distribution leads to larger deviations from the Gaussian case since in this distribution the smaller frequencies which speed up the polarization decay are less weighted than in the other ones.

\section{RTF model including an average over the hopping rates}

The last subsection has shown that an average over distributions of rotation frequencies is not sufficient to reproduce, for the two-frequency model under study, most of the observed anomalies in the longitudinal relaxation rates of glassy systems. We pointed out already in Sec. III B that also an average over different hopping rates should be considered. Namely, the local environments of substances with frozen-in disorder may also be quite different with regard to the dynamics of hopping processes. Therefore, we have performed averages of the depolarization function of the RTF model over rectangular and exponential distributions of the hopping rates, according to Eqs. (13) and (14), respectively. Figure 5 shows the results which we obtained by a fit of the polarization transients with the KWW function (16). The quantities $T_{1}^{-1}$ and $\alpha$ are given as functions of the typical $\gamma$ values, i.e., $\bar{\gamma}=\sqrt{3} \sigma_{\gamma r}$ in the case of the rectangular and $\bar{\gamma}=\sigma_{\gamma e}$ for the exponential distribution. The following features can be recognized.

(i) Generally, the behavior of the relaxation rates as functions of the effective hopping rate is quite similar for both distributions and similar to the behavior of the relaxation where no average over the hopping rates was performed.

(ii) The extra hump in the relaxation rate which is present for the nonaveraged quantity for smaller Larmor frequencies is reduced for the rectangular distribution and almost absent for the exponential distribution.

(iii) The curves are slightly shifted to larger values of the hopping rate and the minimum of $\alpha$ is less pronounced.

(iv) The following frequency dependence can be extracted from the data of Fig. 5 and additional data: rectangular distribution:

exponential distribution:

$$
\begin{array}{lll}
\bar{\gamma}=3 \sqrt{3} \times 10^{3} \mathrm{~s}^{-1} & \rightarrow & T_{1}^{-1} \sim \omega_{0}^{-1.84} \\
\bar{\gamma}=10^{4} \mathrm{~s}^{-1} & \rightarrow & T_{1}^{-1} \sim \omega_{0}^{-1.76}
\end{array}
$$



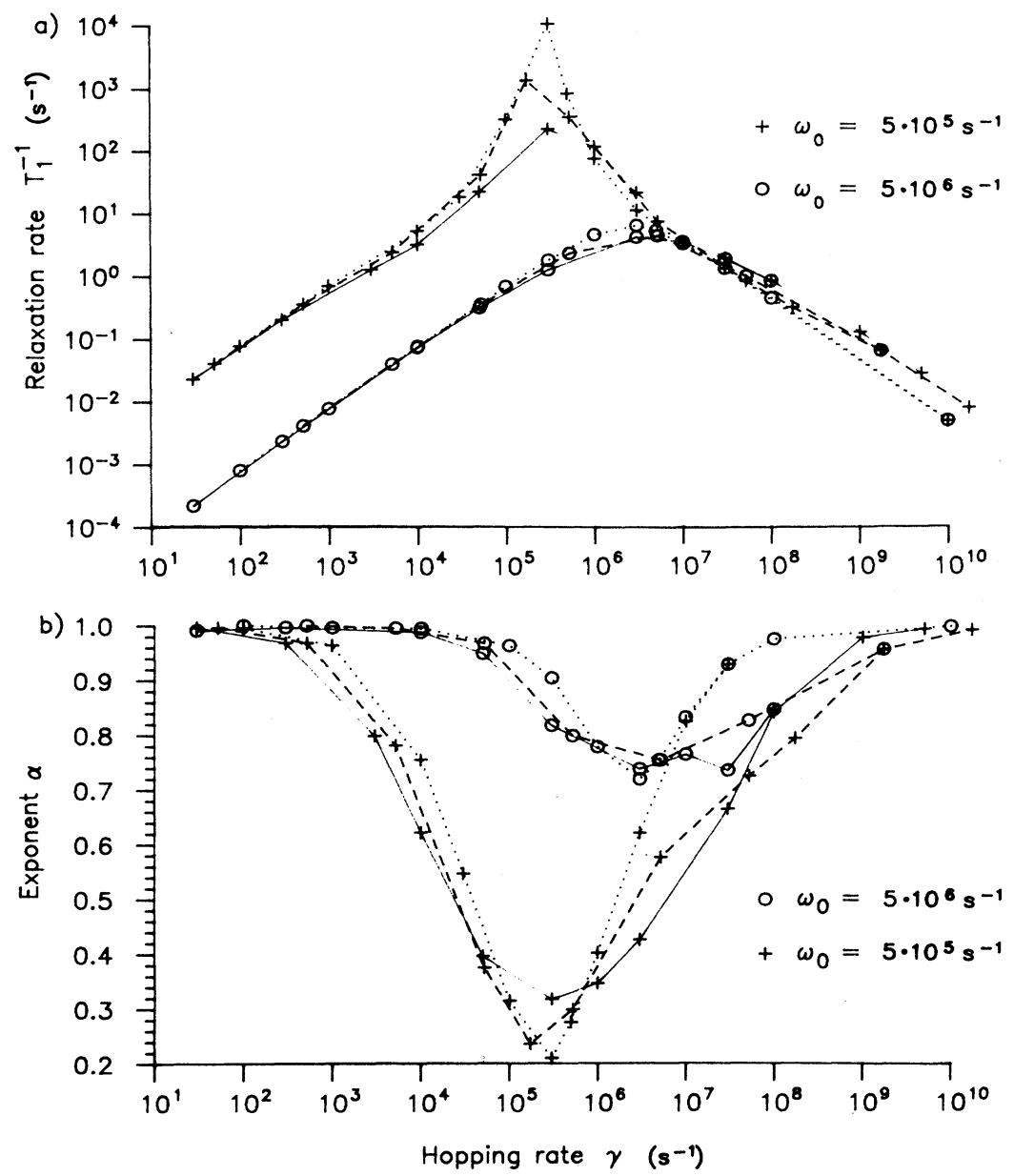

FIG. 5. Plot of the relaxation rate $T_{1}^{-1}$ and of the exponent $\alpha$ vs the hopping rate $\gamma$ for two Larmor frequencies. Here the long-time polarization decay was also averaged over a rectangular distribution (dashed line) and an exponential distribution (solid line) of the hopping rate. For comparison also the result for the nonaveraged hopping rate from Fig. 4 is given (dotted line). (v) Also the curves for the Kohlrausch exponent $\alpha$ are slightly asymmetric in contrast to the case where the hopping rate is not averaged.

In summary, we have to conclude that the additional average over the jump rates only yields a slight modification of the results for the longitudinal relaxation rate and the Kohlrausch exponent which were obtained by the pure frequency average. Accordingly, this average also cannot explain the behavior of the polarization decay in glassy systems.

\section{TIME-DEPENDENT RANDOM TWO-FREQUENCY MODEL}

In this section we introduce an extension of the random two-frequency model. We assume that a spin performs the TF process as described in Sec. II A with the event rate $\gamma$. In addition, we assume that the rotation frequencies of the TF process change according to a Poisson process with the transition rate $\lambda$. Whereas the TF model describes local motion of the spins, the additional renewal process is intended to model possible changes of the environment or transitions of the spins to different local environments. Finally we average over many different realizations of the $\mathrm{TF}$ process, including the renewal process. This leads to the "time-dependent" random twofrequency model.

\section{A. Introduction of the model}

While the frequency changes in real systems may occur more or less gradually, we make the simplifying assumption of the strong-collision model that completely new frequencies are selected at each transition. In detail, the model contains the following assumptions.

(i) In a subsystem (i) the spin motion is described by the TF model which is determined by the jump rate $\gamma$ and the rotation frequencies $\vec{\omega}_{1}^{(i)}, \vec{\omega}_{2}^{(i)}$.

(ii) The spin may change into a new subsystem $(j)$ with the transition rate $\lambda$. The spin motion that takes place there is again given by the TF model with the rotation frequencies $\vec{\omega}_{1}^{(j)}, \vec{\omega}_{2}^{(j)}$, and the same jump rate $\gamma$. A pictorial representation of the process is given in Fig. 6. 


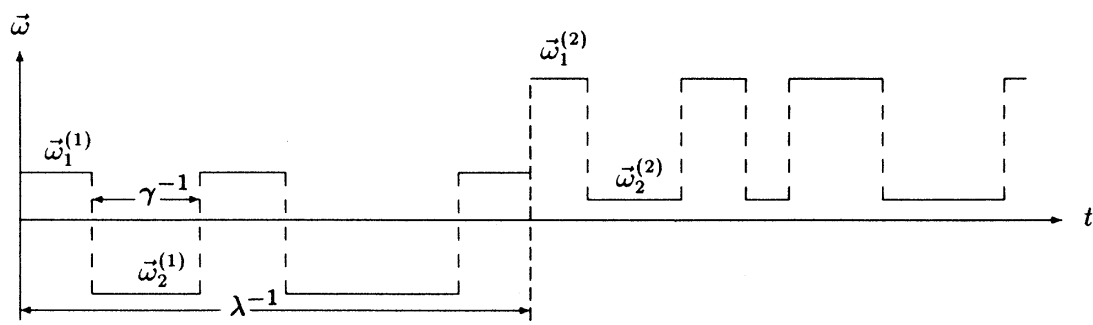

FIG. 6. Schematic representation of the "time-dependent" RTF model. With a transition rate $\lambda$, the hopping process between two sites which are characterized by the rotation frequencies $\vec{\omega}_{1}^{(1)}, \vec{\omega}_{2}^{(1)}$ may change into a hopping process with the frequencies $\vec{\omega}_{1}^{(2)}$, $\vec{\omega}_{2}^{(2)}$. The hopping rate $\gamma$ of the TF process is not changed by this transition. (iii) The transitions to the other subsystems occur corresponding to the strong-collison model $;^{22,27}$ i.e., the frequencies $\vec{\omega}_{1}^{(i)}, \vec{\omega}_{2}^{(i)}$ and ${\overrightarrow{\omega_{1}}}^{(j)},{\overrightarrow{\omega_{2}}}^{(j)}$ are completely uncorrelated.

To derive the spin polarization according to this model, we make a decomposition into the number of transition events, similar to the decomposition into the number of jumps for the TF model,

$$
\vec{P}^{\lambda}(t)=\sum_{l=0}^{\infty} \vec{P}_{l}^{\lambda}(t)
$$

As an example, we give explicitly the term with two transitions,

$$
\vec{P}_{2}^{\lambda}(t)=\lambda^{2} \int_{0}^{t} d t^{\prime} \int_{0}^{t^{\prime}} d t^{\prime \prime} \underline{\underline{P}}^{(k)}\left(t-t^{\prime}\right) \exp \left[-\lambda\left(t-t^{\prime}\right)\right] \underline{\underline{P}}^{(j)}\left(t^{\prime}-t^{\prime \prime}\right) \exp \left[-\lambda\left(t^{\prime}-t^{\prime \prime}\right)\right] \underline{\underline{P}}^{(i)}\left(t^{\prime \prime}\right) \exp \left(-\lambda t^{\prime \prime}\right) \cdot \overrightarrow{S_{0}}
$$

Here the quantity $\underline{\underline{P}}^{(i)}(t)$ is the solution of the TF model for a given pair of frequencies $\vec{\omega}_{1}^{(i)}$, $\vec{\omega}_{2}^{(i)}$, i.e., the result of Sec. II A. By making this identification we assume that we have already replaced particular two-frequency processes within the sojourn at fixed frequencies by the ensemble average over all processes for given frequency pairs. This is justified by the following consideration: We consider large systems which comprise many different subsystems with fixed frequencies and the ensemble average over many transition processes of the spins, both within the subsystems and between the subsystems. The average over the processes within the subsystems justifies the use of the $P^{(i)}(t)$ in Eq. (22) and all other terms, averaging over the transitions between subsystems leads to an ensemble average over many different realizations of the disorder. If we assume that all pairs of rotation frequencies $\vec{\omega}_{1}^{(i)}, \vec{\omega}_{2}^{(i)}$ are statistically independent, and taken from the same probability distribution, we can replace the $\underline{\underline{P}}^{(i)}(t)$ in the terms of the series (21) by the average, $\left\{\underline{\underline{\tilde{P}}}^{\gamma}\right\}_{\vec{\omega}_{1}, \vec{\omega}_{2}}$, i.e., by the result for the RTF model.

After the replacement, the term that describes two transitions between different frequencies reads

$$
\begin{aligned}
\left\{\vec{P}^{\lambda}(t)\right\}_{\vec{\omega}_{1}, \overrightarrow{\omega_{2}}}= & \lambda^{2} \int_{0}^{t} d t^{\prime} \int_{0}^{t^{\prime}} d t^{\prime \prime}\left\{\underline{\underline{\tilde{P}}}^{\gamma}\right\}_{\vec{\omega}_{1}, \vec{\omega}_{2}}\left(t-t^{\prime}\right) \exp \left[-\lambda\left(t-t^{\prime}\right)\right] \\
& \times\left\{\underline{\underline{\tilde{P}}}^{\gamma}\right\}_{\overrightarrow{\omega_{1}, \vec{\omega}_{2}}}\left(t^{\prime}-t^{\prime \prime}\right) \exp \left[-\lambda\left(t^{\prime}-t^{\prime \prime}\right)\right]\left\{\underline{\underline{\tilde{P}}}^{\gamma}\right\}_{\vec{\omega}_{1}, \vec{\omega}_{2}}\left(t^{\prime \prime}\right) \exp \left(-\lambda t^{\prime \prime}\right) \cdot \overrightarrow{S_{0}}
\end{aligned}
$$

In the Laplace domain, one has a triple product of terms, with a shifted Laplace variable $s+\lambda$. After this transformation a geometric series is found which is easily summed up. The result is

$$
\left\{\tilde{\vec{P}}^{\lambda}(s)\right\}_{\vec{\omega}_{1}, \vec{\omega}_{2}}=\left\{\underline{\underline{\underline{P}}}^{\gamma}(s+\lambda)\right\}_{\vec{\omega}_{1}, \vec{\omega}_{2}}\left(\underline{\underline{1}}-\lambda\left\{\underline{\underline{\tilde{P}}}^{\gamma}(s+\lambda)\right\}_{\vec{\omega}_{1}, \vec{\omega}_{2}}\right)^{-1} \cdot \overrightarrow{S_{0}} .
$$

As can be shown explicitly ${ }^{24}$ the longitudinal polarization develops independently of the polarization components in the $x-y$ subspace; provided that the $x$ and $y$ components of the frequencies $\vec{\omega}_{1}$ and $\vec{\omega}_{2}$ are equally distributed around zero. This condition is fulfilled, for instance, for the Gaussian distribution (10) with a longitudinal Zeeman field $\left(\left\langle\omega_{x}\right\rangle=\left\langle\omega_{y}\right\rangle=0 \leq\left\langle\omega_{z}\right\rangle\right)$. In view of the decoupling of the longitudinal and transverse components the longitudinal polarization, $\left\{\tilde{P}_{z}^{\lambda}(s)\right\}_{\vec{\omega}_{1}, \vec{\omega}_{2}}$, reduces to

$$
\left\{\tilde{P}_{z}^{\lambda}(s)\right\}_{\vec{\omega}_{1}, \vec{\omega}_{2}}=\left\{\underline{\underline{P}}_{z z}^{\gamma}(s+\lambda)\right\}_{\vec{\omega}_{1}, \vec{\omega}_{2}}\left(1-\lambda\left\{\underline{\underline{P}}_{z z}^{\gamma}(s+\lambda)\right\}_{\vec{\omega}_{1}, \vec{\omega}_{2}}\right)^{-1},
$$

with the initial condition $\overrightarrow{S_{0}}=\vec{e}_{z}$.

A further analytical investigation of Eq. (25) seems not possible since no explicit solution of the averaged polarization 
$\left\{\underline{\underline{P}}^{\gamma}(s)\right\}_{\vec{\omega}_{1}, \vec{\omega}_{2}}$ is known. Therefore one is restricted to numerical means for getting further information about the polarization decay in the time-dependent RTF model. Corresponding to Eq. (7) the relevant term $\underline{\underline{P}}_{z z}^{\gamma}(s+\lambda)$ in $(25)$ can be represented in the form

$$
\underline{\tilde{P}}_{z z}^{\gamma}(s+\lambda)=\frac{1}{2}\left[\sum_{i=0}^{5} \tilde{a}_{i}\left(\vec{\omega}_{1}, \vec{\omega}_{2} ; \gamma, \lambda\right) s^{i}\right]\left[\sum_{j=0}^{6} \tilde{b}_{j}\left(\vec{\omega}_{1}, \vec{\omega}_{2} ; \gamma, \lambda\right) s^{j}\right]^{-1}
$$

with $\lambda$-dependent coefficients that are listed in the Appendix.

\section{B. Behavior at intermediate time scales}

Corresponding to the discussion of the simple RTF model we investigate the behavior of the "timedependent" RTF model on intermediate time scales. Figure 7 presents the results of the numerical evaluation of Eq. (25) where the matrix term $\underline{\underline{\tilde{P}}}_{z z}^{\gamma}(s+\lambda)(26)$ is averaged over a Gaussian frequency distribution $(\sigma=1$ and $\vec{\omega}_{0}=0$ ) with fixed hopping rate $\gamma=50$.

Obviously, and as expected, the polarization decay of the extended model corresponds to the one found for the RTF model in the limit of small transition rates $\lambda$ between different subsystems. An increase of this quantity subsequently leads to the vanishing of the characteristic minimum of the Kubo-Toyabe function. The resulting behavior resembles more and more the polarization decay of the "strong-collision" model in the case of $\lambda \geq \gamma$, as it is seen by comparison with the results from Ref. 27 which are also plotted in Fig. 7.

By comparing Fig. 2 and Fig. 7 we can recognize a "competition" between the motional-narrowing effect of the RTF model for large hopping rates $\gamma$ and the motional-narrowing effect of the strong-collision model caused by rapid transitions between distinct subsystems.

\section{Asymptotic polarization decay for the "time-dependent" RTF model}

Finally, we investigate this extension with regard to its long-time decay characteristic. As in Sec. IV A we utilize the approximation (17) where now the quantity $\left\{\underline{\tilde{P}}_{z z}^{\gamma}(s+\right.$ $\lambda)\}$ is expressed in terms of the $\lambda$-dependent coefficients $\tilde{a}_{i}, \tilde{b}_{j}$; see the Appendix. The expression is then averaged over a Gaussian distribution of the frequencies $\vec{\omega}_{1}, \vec{\omega}_{2}$. Now we have to deal with two event rates; we regard the processes to be thermally activated, and described by an Arrhenius ansatz,

$$
\gamma=\gamma_{0} \exp \left(-\frac{E_{\gamma}}{k_{B} T}\right), \quad \lambda=\lambda_{0} \exp \left(-\frac{E_{\lambda}}{k_{B} T}\right) .
$$

Here $E_{\lambda, \gamma}$ designate the activation energies of the processes and $\gamma_{0}, \lambda_{0}$ are usually called attempt frequencies. Relating the parameters of the transition rate $\lambda$ to the ones of the hopping rate $\gamma$ by $\lambda_{0}=b \gamma_{0}$ and $E_{\lambda}=a E_{\gamma}$, we have the scaling relation

$$
\lambda=\frac{b}{\gamma_{0}^{a-1}} \gamma^{a} \text {. }
$$

At this point we have to keep in mind that $\gamma \leq \gamma_{0}$ and $\lambda \leq \lambda_{0}$.

In a first run, we started with hopping rates $\gamma$ that are suggested by typical experiments, ${ }^{34}$ namely, $E_{\gamma}=1$ $\mathrm{eV}$, and an attempt frequency which lies in the range $\gamma_{0}=10^{11}-10^{13} \mathrm{~s}^{-1}$. We then assumed that the activation energy $E_{\lambda}$ of the transition process is $2 E_{\gamma}$ or $1.5 E_{\gamma}$, corresponding to $a=2$ or $a=1.5$, respectively. We further assumed that $\lambda_{0}=\gamma_{0}$ or $b=1$. We selected three combinations of parameters for a fixed value of the Larmor frequency $\omega_{0}=5 \times 10^{6} \mathrm{~s}^{-1}$. The results for the longitudinal relaxation rates $T_{1}^{-1}$ and for the Kohlrausch

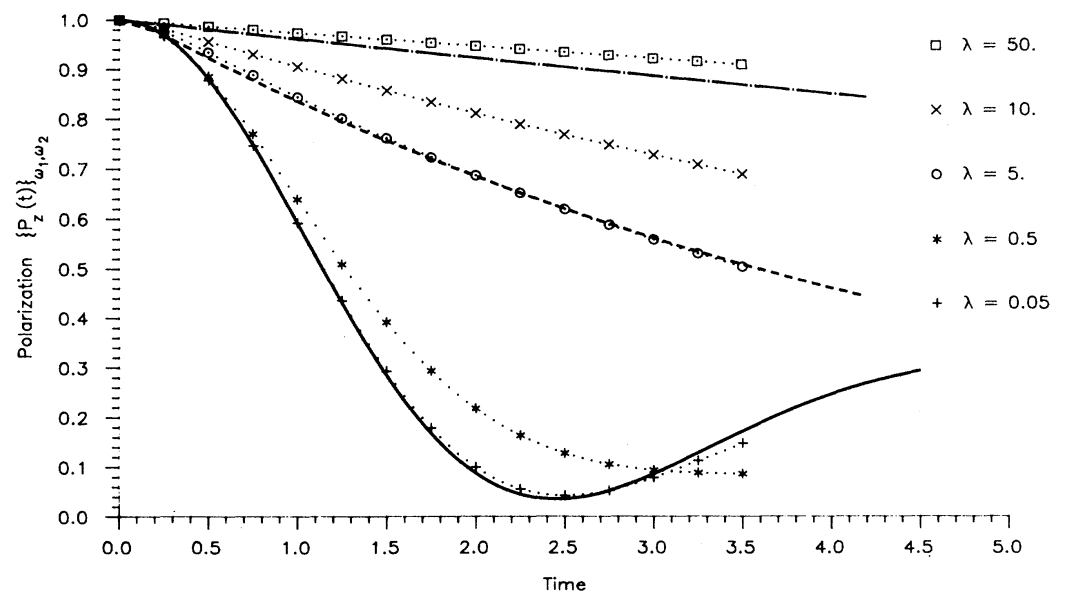

FIG. 7. Representation of the averaged polarization of the "time-dependent" RTF model Eq. (25) as a function of time, for fixed hopping rate $\gamma=50$. The average over the polarization $\underline{\underline{\hat{P}}}_{z z}^{\gamma}(s+\lambda)$, Eq. (26),

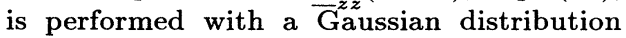
with $\sigma=1$ and $\vec{\omega}_{0}=0$. The dotted lines serve as visual support. The solid line represents the corresponding solution (10) of the RTF model in the limit $\gamma \rightarrow \infty$, whereas the dashed (dash-dotted) line corresponds to the "strong-collision" model of Hayano et al. (Ref. 27) for $\lambda=10(\lambda=50)$ with fixed $\gamma=0$. The time is given in units of the inverse of a reference hopping rate $\gamma$. 
exponent $\alpha$ are given in Fig. 8, together with the result for zero transition rate. The following features are recognized.

(i) The curves for $T_{1}^{-1}$ exhibit now two maxima, instead of one.

(ii) Two of the curves for the exponent $\alpha$ show a less pronounced minimum than in the case $\lambda=0$.

If one regards the actual values of $\lambda$ as a function of the hopping rate $\gamma$, one sees that $\lambda$, for smaller $\gamma$, is generally much smaller than $\gamma$. This explains that the behavior of $T_{1}^{-1}$ in the region of the first maximum is similar to the case $\lambda=0$. The second maximum corresponds to rates $\lambda$ of the order of the Larmor frequency. This means that the transition process leads to a second, independent maximum in the relaxation rate, for the time-dependent RTF model. The behavior of the exponent $\alpha$ for the parameter choice (A) is similar to the case $\lambda=0$. For this choice of parameters, $\lambda$ is very small, except at the right end of the abscissa. Evidently an increase of the transition rate $\lambda$ leads to a smoothing out of the behavior of $\alpha$ as a function of the hopping rate $\gamma$.

In a second run, we avoided the appearance of two distinct maxima which are caused by the independence of the hopping from the transition process. For this purpose we choose the parameters in such a way that both maxima coincide. This will occur when $\lambda$ becomes approximately equal to $\gamma$ at the Larmor frequency $\omega_{0}$, $\lambda \simeq \gamma \simeq \omega_{0}$. For this purpose one has to assume a large attempt frequency $\lambda_{0}$, i.e., $b \gg 1$. We leave the question open whether such a choice is physically reasonable. We took the scale factor $a=1.5$ or $a=2, b=10^{4}$, and studied three sets of parameters; see Fig. 9. Two different values of the Larmor frequency were taken, to fulfill the condition $\lambda \simeq \gamma \simeq \omega_{0}$ near the maximum.

The results for the longitudinal relaxation rate $T_{1}^{-1}$ in Fig. 9 show the expected single maximum, but this has been achieved by force. Not expected is the rise of $T_{1}^{-1}$ for large values of $\gamma$. The interpretation of this rise is unclear; however, it occurs for values of $\lambda$ which are unphysically large. The slopes of the curves for $T_{1}^{-1}$ as functions of $\gamma$ differ on both sides of the maxima. At the low-temperature side, the slope is essentially determined by the activation energy of the hopping process, while the slope on the high-temperature side is determined by the (higher) activation energy of the transition process. This leads to an asymmetry of the $T^{-1}$ curves showing qualitatively the same trend as observed in most experiments. The frequency dependence on the low-temperature side is quite similar to the usual frequency dependence of the BPP theory; for instance, for case (D)
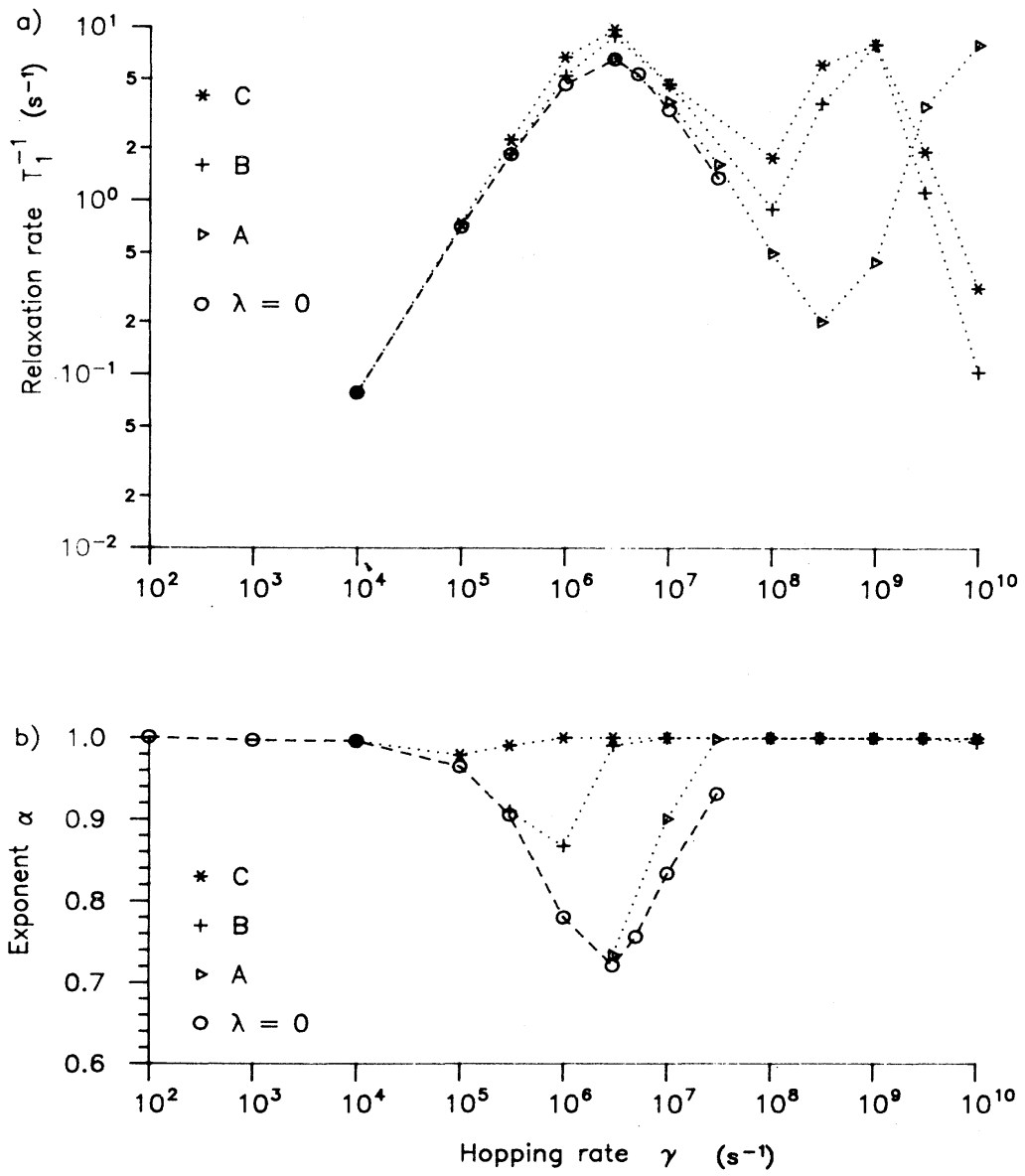

FIG. 8. Plot of the relaxation rate $T_{1}^{-1}$ and of the exponent $\alpha$ for the case of the "time-dependent" RTF model for three combinations of the jump and the transition rates: (A) $\lambda=10^{-13} \gamma^{2} \mathrm{~s}$, (B) $\lambda=10^{-11} \gamma^{2} \mathrm{~s}$, (C) $\lambda=10^{-13 / 2} \gamma^{3 / 2} \mathrm{~s}^{1 / 2}$. The Zeeman field is set to $\omega_{0}=5 \times 10^{6} \mathrm{~s}^{-1}$. The dashed line for $\lambda=0$ is taken from Fig. 4 . 


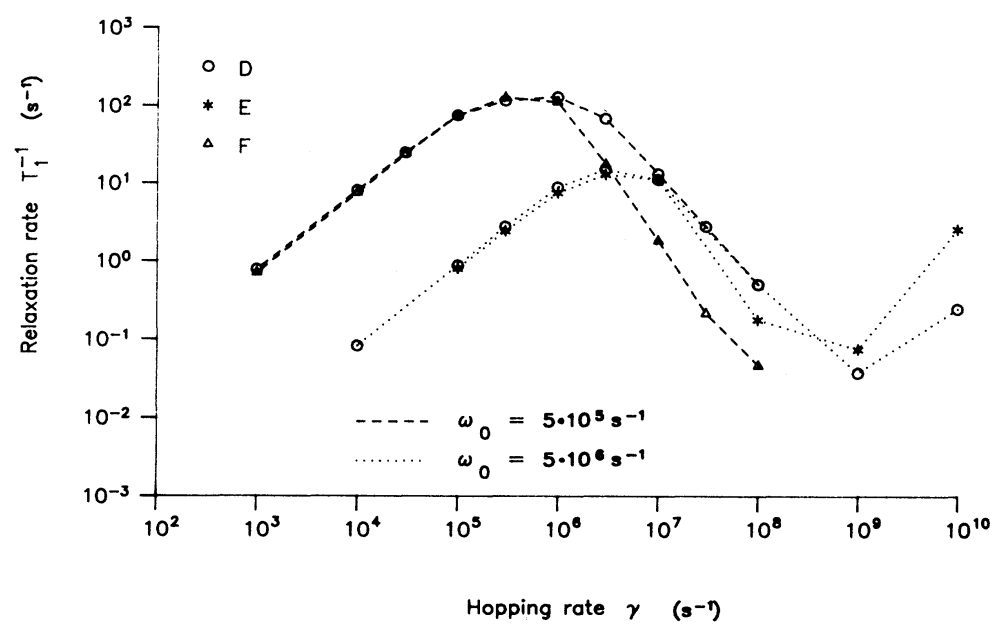

FIG. 9. Plot of the relaxation rate $T_{1}^{-1}$, as in Fig. 8, for the parameter values (D) $\lambda=10^{-7 / 2} \gamma^{3 / 2} \mathrm{~s}^{1 / 2}$, (E) $\lambda=10^{-7} \gamma^{2} \mathrm{~s},(\mathrm{~F})$ $\lambda=10^{-6} \gamma^{2}$ s.

$$
\gamma=10^{3} \mathrm{~s}^{-1} \longrightarrow T_{1}^{-1} \sim \omega_{0}^{-1.99}
$$

In this respect the result of the time-dependent RTF model is somewhat disappointing.

No results for the Kohlrausch exponent are given in Fig. 9. In fact, this exponent is now practically one for all values of $\gamma$ and $\omega_{0}$. This is a consequence of the large transition rates $\lambda$; cf. the disappearance of the minimum for larger $\lambda$ in Fig. 7. To summarize, the accomplishment of the time-dependent RTF model seems to be a potential explanation of asymmetric rate maxima. However, this is achieved at the expense of parameters with the undesirable property that now the transition rates exceed the values of the hopping rates, on the hightemperature side. This is in contradiction to the underlying idea that spin motion within each subsystem shall be faster than the transition processes between the different subsystems. Further comments on this problem will be given in the Conclusion.

\section{CONCLUSION}

In this paper we were concerned with a derivation of spin relaxation in disordered systems. The underlying postulate of our theory is that one should perform an inhomogeneous average also for dynamical situations in systems with quenched disorder. In such systems one has very different local environments where probe spins may behave quite differently. The dynamics of these probe spins has to be treated first for these local environments; thereafter, the result has to be averaged over the different configurations. Previous work did implement this program by averaging exponential decay functions, $\exp \left(-t / T_{1,2}\right)$, over distributions of the relaxation times. Our intention was to use models for the local motions that are applicable at all times scales. Hence we exemplified our approach on simple stochastic models, in particular on a two-frequency model which involves motion of the probe spins over two sites. We also introduced a time-dependent two-frequency model which takes time-dependent changes of the local environment into account.

Our efforts were only partially successful. The random two-frequency model yields a behavior on intermediate time scales which is in qualitative agreement with recent $\mu \mathrm{SR}$ experiments in $\mathrm{NbTi}$. In this case the local disorder is relatively well understood. The spin relaxation of the random two-frequency model shows nonexponential relaxation at long times, which may be fitted by the Kohlrausch-Williams-Watts function. One interesting result is a temperature- and field-dependent Kohlrausch exponent; such a behavior has been observed in recent experiments. The behavior of the associated longitudinal spin relaxation rate does not exhibit the characteristic features of glassy systems. That is, a BPP plot of the relaxation rate does not show asymmetry or modified exponents of the frequency dependence. An asymmetry of the BPP plot could be achieved for the time-dependent extension of the random two-frequency model; however, at high temperatures the required renewal rate becomes larger than the rates of the motional processes within each subsystem.

The conclusion is that the models which were considered in this paper are still too naive in comparison with actual glassy substances. The restriction of the motion of probe spins to two sites may be too simple, and one should consider more complicated local motional processes. There are several other possibilities to extend the present approach. For instance, one may take dipolar or other interactions into account by treating the local dynamics by the methods of Ref. 18 and then performing inhomogeneous averages. Also the extension of the "time-dependent" two-frequency model should be further elaborated, since it contains, in principle, the transition from restricted to spatially unrestricted dynamics.

\section{ACKNOWLEDGMENTS}

Helpful discussions with W. Puin and A. Schirmer are gratefully acknowledged. This work was supported by the DFG through SFB 173 and by the BMFT. 


\section{APPENDIX}

First we list the coefficients $a_{i}$ and $b_{j}$ as they are obtained from Eq. (4) by algebraic formula manipulation:

$$
\begin{aligned}
a_{0}\left(\vec{\omega}_{1}, \vec{\omega}_{2}, \gamma\right) & =4 \gamma^{3}\left(\omega_{1 z}+\omega_{2 z}\right)^{2}+\gamma\left(\omega_{1 z} \vec{\omega}_{2}+\omega_{2 z} \vec{\omega}_{1}\right)^{2} \\
a_{1}\left(\vec{\omega}_{1}, \vec{\omega}_{2}, \gamma\right), & =2 \gamma^{2}\left(\vec{\omega}_{1}-\vec{\omega}_{2}\right)^{2}+8 \gamma^{2}\left(\omega_{1 z}+\omega_{2 z}\right)^{2}-8 \gamma^{2} \omega_{1 z} \omega_{2 z}+\omega_{1 z}^{2} \vec{\omega}_{2}^{2}+\omega_{2 z}^{2} \vec{\omega}_{1}^{2}, \\
a_{2}\left(\vec{\omega}_{1}, \vec{\omega}_{2}, \gamma\right) & =16 \gamma^{3}+5 \gamma\left(\omega_{1 z}+\omega_{2 z}\right)^{2}-8 \gamma \omega_{1 z} \omega_{2 z}+2 \gamma\left(\vec{\omega}_{1}^{2}+\vec{\omega}_{2}^{2}\right)+\gamma\left(\vec{\omega}_{1}-\vec{\omega}_{2}\right)^{2}, \\
a_{3}\left(\vec{\omega}_{1}, \vec{\omega}_{2}, \gamma\right) & =24 \gamma^{2}+\vec{\omega}_{1}^{2}+\vec{\omega}_{2}^{2}+\omega_{1 z}^{2}+\omega_{2 z}^{2}, \\
a_{4}\left(\vec{\omega}_{1}, \vec{\omega}_{2}, \gamma\right) & =12 \gamma \\
a_{5}\left(\vec{\omega}_{1}, \vec{\omega}_{2}, \gamma\right) & =2 \\
b_{0}\left(\vec{\omega}_{1}, \vec{\omega}_{2}, \gamma\right) & =\gamma^{2}\left[\vec{\omega}_{1}^{2} \vec{\omega}_{2}^{2}-\left(\vec{\omega}_{1} \cdot \vec{\omega}_{2}\right)^{2}\right] \\
b_{1}\left(\vec{\omega}_{1}, \vec{\omega}_{2}, \gamma\right) & =2 \gamma, \vec{\omega}_{1}^{2} \vec{\omega}_{2}^{2}+2 \gamma^{3}\left(\vec{\omega}_{1}+\vec{\omega}_{2}\right)^{2} \\
b_{2}\left(\vec{\omega}_{1}, \vec{\omega}_{2}, \gamma\right) & =\vec{\omega}_{1}^{2} \vec{\omega}_{2}^{2}+2 \gamma^{2} \vec{\omega}_{1} \cdot \vec{\omega}_{2}+5 \gamma^{2}\left(\vec{\omega}_{1}^{2}+\vec{\omega}_{2}^{2}\right), \\
b_{3}\left(\vec{\omega}_{1}, \vec{\omega}_{2}, \gamma\right) & =8 \gamma^{3}+4 \gamma\left(\vec{\omega}_{2}^{2}+\vec{\omega}_{1}^{2}\right), \\
b_{4}\left(\vec{\omega}_{1}, \vec{\omega}_{2}, \gamma\right) & =12 \gamma^{2}+\vec{\omega}_{1}^{2}+\vec{\omega}_{2}^{2}, \\
b_{5}\left(\vec{\omega}_{1}, \vec{\omega}_{2}, \gamma\right) & =6 \gamma \\
b_{6}\left(\vec{\omega}_{1}, \vec{\omega}_{2}, \gamma\right) & =1 .
\end{aligned}
$$

The $\lambda$-dependent coefficients $\tilde{a}_{i}$ and $\tilde{b}_{j}$ that are needed in Eq. (26) are given by

$$
\begin{aligned}
\tilde{a}_{0}\left(\vec{\omega}_{1}, \vec{\omega}_{2}, \gamma, \lambda\right) & =g_{0}+\varepsilon g_{1}+\varepsilon^{2} g_{2}+\varepsilon^{3} g_{3}+\varepsilon^{4} g_{4}+\varepsilon^{5} g_{5} \\
\tilde{a}_{1}\left(\vec{\omega}_{1}, \vec{\omega}_{2}, \gamma, \lambda\right) & =g_{1}+2 \varepsilon g_{2}+3 \varepsilon^{2} g_{3}+4 \varepsilon^{3} g_{4}+5 \varepsilon^{4} g_{5} \\
\tilde{a}_{2}\left(\vec{\omega}_{1}, \vec{\omega}_{2}, \gamma, \lambda\right) & =g_{2}+3 \varepsilon g_{3}+6 \varepsilon^{2} g_{4}+10 \varepsilon^{3} g_{5} \\
\tilde{a}_{3}\left(\vec{\omega}_{1}, \vec{\omega}_{2}, \gamma, \lambda\right) & =g_{3}+4 \varepsilon g_{4}+10 \varepsilon^{2} g_{5} \\
\tilde{a}_{4}\left(\vec{\omega}_{1}, \vec{\omega}_{2}, \gamma, \lambda\right) & =g_{4}+5 \varepsilon g_{5} \\
\tilde{a}_{5}\left(\vec{\omega}_{1}, \vec{\omega}_{2}, \gamma, \lambda\right) & =g_{5} \\
\tilde{b}_{0}\left(\vec{\omega}_{1}, \vec{\omega}_{2}, \gamma, \lambda\right) & =h_{0}+\varepsilon^{2} h_{2}+\varepsilon^{4} h_{4}+\varepsilon^{6} \\
& =\left(\varepsilon^{2}-\gamma^{2}\right)^{3}+\varepsilon^{2}\left[\vec{\omega}_{1}^{2} \vec{\omega}_{2}^{2}-\gamma^{2}\left(\vec{\omega}_{1}-\vec{\omega}_{2}\right)^{2}\right]+\varepsilon^{4}\left(\vec{\omega}_{1}^{2}+\vec{\omega}_{2}^{2}\right)-\gamma^{2}\left(\vec{\omega}_{1} \cdot \vec{\omega}_{2}\right)^{2}-2 \gamma^{4} \vec{\omega}_{1} \cdot \vec{\omega}_{2}, \\
& =2 \varepsilon\left[\vec{\omega}_{1}^{2} \vec{\omega}_{2}^{2}-\gamma^{2}\left(\vec{\omega}_{1}-\vec{\omega}_{2}\right)^{2}+3\left(\varepsilon^{2}-\gamma^{2}\right)^{2}+2 \varepsilon^{2}\left(\vec{\omega}_{1}^{2}+\vec{\omega}_{2}^{2}\right)\right] \\
\tilde{b}_{1}\left(\vec{\omega}_{1}, \vec{\omega}_{2}, \gamma, \lambda\right) & =2 \varepsilon\left[h_{2}+2 \varepsilon^{2} h_{4}+3 \varepsilon^{4}\right] \\
\tilde{b}_{2}\left(\vec{\omega}_{1}, \vec{\omega}_{2}, \gamma, \lambda\right) & =h_{2}+6 \varepsilon^{2} h_{4}+15 \varepsilon^{4} \\
\tilde{b}_{3}\left(\vec{\omega}_{1}, \vec{\omega}_{2}, \gamma, \lambda\right) & =4 \varepsilon h_{4}+20 \varepsilon^{3} \\
\tilde{b}_{4}\left(\vec{\omega}_{1}, \vec{\omega}_{2}, \gamma, \lambda\right) & =h_{4}+15 \varepsilon^{2} \\
\tilde{b}_{5}\left(\vec{\omega}_{1}, \vec{\omega}_{2}, \gamma, \lambda\right) & =6 \varepsilon \\
\tilde{b}_{6}\left(\vec{\omega}_{1}, \vec{\omega}_{2}, \gamma, \lambda\right) & =1
\end{aligned}
$$

where we have defined $\varepsilon=\gamma+\lambda .^{24}$ The coefficients $g_{i}, h_{j}$ are given by

$$
\begin{aligned}
& g_{0}\left(\vec{\omega}_{1}, \vec{\omega}_{2} ; \gamma\right)=2 \gamma^{5}+2 \gamma^{3}\left(\vec{\omega}_{1} \cdot \vec{\omega}_{2}+\omega_{1 z} \omega_{2 z}\right)+2 \gamma \vec{\omega}_{1} \cdot \vec{\omega}_{2} \omega_{1 z} \omega_{2 z}, \\
& g_{1}\left(\vec{\omega}_{1}, \vec{\omega}_{2} ; \gamma\right)=2 \gamma^{4}-\gamma^{2}\left[\vec{\omega}_{1}^{2}+\vec{\omega}_{2}^{2}-\left(\omega_{1 z}+\omega_{2 z}\right)^{2}-2 \omega_{1 z} \omega_{2 z}\right]+\vec{\omega}_{1}^{2} \omega_{2 z}^{2}+\vec{\omega}_{2}^{2} \vec{\omega}_{1 z}^{2}, \\
& g_{2}\left(\vec{\omega}_{1}, \vec{\omega}_{2} ; \gamma\right)=-4 \gamma^{3}-2 \gamma\left[\vec{\omega}_{1} \cdot \vec{\omega}_{2}-\left(\omega_{1 z}+\omega_{2 z}\right)^{2}+\omega_{1 z} \omega_{2 z}\right], \\
& g_{3}\left(\vec{\omega}_{1}, \vec{\omega}_{2} ; \gamma\right)=-4 \gamma^{2}+\vec{\omega}_{1}^{2}+\vec{\omega}_{2}^{2}+\omega_{1 z}^{2}+\omega_{2 z}^{2}, \\
& g_{4}\left(\vec{\omega}_{1}, \vec{\omega}_{2} ; \gamma\right)=2 \gamma \\
& g_{5}\left(\vec{\omega}_{1}, \vec{\omega}_{2} ; \gamma\right)=2 \\
& h_{0}\left(\vec{\omega}_{1}, \vec{\omega}_{2} ; \gamma\right)=-\gamma^{2}\left(\gamma^{2}+\vec{\omega}_{1} \cdot \vec{\omega}_{2}\right)^{2}, \\
& h_{2}\left(\vec{\omega}_{1}, \vec{\omega}_{2} ; \gamma\right)=3 \gamma^{4}-\gamma^{2}\left(\vec{\omega}_{2}-\vec{\omega}_{1}\right)^{2}+\vec{\omega}_{1}^{2} \vec{\omega}_{2}^{2}, \\
& h_{4}\left(\vec{\omega}_{1}, \vec{\omega}_{2} ; \gamma\right)=-3 \gamma^{2}+\vec{\omega}_{1}^{2}+\vec{\omega}_{2}^{2}, \\
& h_{6}\left(\vec{\omega}_{1}, \vec{\omega}_{2} ; \gamma\right)=1 .
\end{aligned}
$$


${ }^{1}$ H. Ackermann, P. Heitjans, and H.-J. Stöckmann, in $H y$ perfine Interactions of Radioactive Nuclei, edited by $\mathrm{J}$. Christiansen, Topics in Current Physics Vol. 31 (Springer, Berlin, 1983), p. 291.

${ }^{2}$ P. Heitjans, Solid State Ion. 18 \& 19, 50 (1986).

${ }^{3}$ A. Schenck, Muon Spin Rotation Spectroscopy: Principles and Applications in Solid State Physics (Hilger, Bristol, 1985).

4 C.P. Slichter, Principles of Magnetic Resonance, 3rd ed. (Springer, Berlin, 1990).

5 A. Abragam, Principles of Nuclear Magnetism (Oxford University Press, London, 1961).

${ }^{6}$ N. Bloembergen, E.M. Purcell, and R.V. Pound, Phys. Rev. 73, 679 (1948).

${ }^{7}$ R.K. Wangsness and F. Bloch, Phys. Rev. 89, 728 (1953); F. Bloch, ibid. 102, 104 (1956).

${ }^{8}$ A.G. Redfield, IBM J. Res. Dev. 1, 19 (1957).

${ }^{9}$ H.-J. Stöckmann and P. Heitjans, J. Non-Cryst. Solids 66, 501 (1984).

${ }^{10}$ E. Roessler, M. Taupitz, and H.M. Vieth, J. Phys. Chem. 94, 6879 (1990).

${ }^{11}$ W. Schnauss, F. Fujara, K. Hartmann, and H. Sillescu, Chem. Phys. Lett. 166, 381 (1990).

${ }^{12}$ W. Schnauss, F. Fujara, and H. Sillescu, J. Chem. Phys. 97, 1378 (1992).

${ }^{13}$ R. Geil and G. Hinze, Chem. Phys. Lett. 216, 51 (1993).

${ }^{14}$ P.W. Anderson, J. Phys. Soc. Jpn. 9, 316 (1954).

${ }^{15}$ R. Kubo and K. Tomita, J. Phys. Soc. Jpn. 9, 888 (1954).

${ }^{16} \mathrm{R}$. Kubo and T. Toyabe, in Magnetic Resonance and Relaxation, Proceedings of the XIVth Colloque Ampère, Ljubljana, 1966, edited by R. Blinc (North-Holland, Amsterdam, 1967), p. 810.

${ }^{17}$ M. Celio, Phys. Rev. Lett. 56, 2720 (1986).

${ }^{18}$ H.-J. Stöckmann, J. Phys. Condens. Matter 1, 5101 (1989).

${ }^{19}$ G. Diezemann and W. Schirmacher, J. Phys. Condens.
Matter 2, 6681 (1990).

${ }^{20}$ P. Heitjans, A. Körblein, H. Ackermann, D. Dubbers, F. Fujara, and H.J. Stöckmann, J. Phys. F 15, 41 (1985).

${ }^{21}$ P. Borgs, K.W. Kehr, and P. Heitjans, Phys. Lett. A 155, 429 (1991).

${ }^{22}$ K.W. Kehr, G. Honig, and D. Richter, Z. Phys. B 32, 49 (1978).

${ }^{23}$ Handbook of Mathematical Functions, 9th ed., edited by M. Abramowitz and I.A. Stegun (Dover, New York, 1970).

${ }^{24}$ P. Borgs, Ph.D. thesis, Universität Hannover, 1993.

${ }^{25}$ J.H. Van Vleck, Phys. Rev. 74, 1168 (1948).

${ }^{26}$ G. Honig and U. Hirdes, J. Comput. Appl. Math. 10, 113 (1984).

${ }^{27}$ R.S. Hayano, Y.J. Uemura, J. Imazato, N. Nishida, T. Yamazaki, and R. Kubo, Phys. Rev. B 20, 850 (1979).

${ }^{28}$ H.-J. Fell, R. Hempelmann, O. Hartmann, S. Harris, and R. Wäppling, Ber. Bunsenges. Phys. Chem. 95, 1091 (1991).

${ }^{29}$ A. Magerl, A.J. Dianoux, H. Wipf, K. Neumaier, and I.S. Anderson, Phys. Rev. Lett. 56, 159 (1986).

${ }^{30}$ A.M. Stoneham, Phys. Lett. 94A, 353 (1983).

${ }^{31}$ W.F. Lankford, H.K. Birnbaum, A.T. Fiory, R.P. Minnich, K.G. Lynn, C.E. Stronach, L.H. Bieman, W.J. Kossler, and J. Lindemuth, Hyperfine Interact. 4, 833 (1978).

${ }^{32}$ K.L. Ngai, Solid State Ion. 5, 27 (1981).

${ }^{33}$ K. Funke and R. Hoppe, Solid State Ion. 40 \& 41, 200 (1990).

34 P. Heitjans, W. Faber, and A. Schirmer, J. Non-Cryst. Solids 131-133, 1053 (1991).

35 The appearance of two real roots is associated with the eventual exponential decay of the plateau value $(1 / 3)$ of the Kubo-Toyabe behavior for finite transition rates.

${ }^{36}$ In this context we remark that the Larmor frequencies in NMR measurements typically exceed the value $10^{6} \mathrm{~s}^{-1}$.

${ }^{37}$ G. Hinze, Ph.D. thesis, Universität Mainz, 1993.

${ }^{38}$ W. Franke, Diploma thesis, Universität Hannover, 1990. 